\begin{tabular}{|c|c|}
\hline $\begin{array}{l}\text { MAY } 191997 \% \text { ENGINEERING DATA TRANSMITTAL } \\
\text { Sta, } 37 \text {; }\end{array}$ & 1. EDT $\quad 6212^{\text {Pase } 1 \text { of }} \frac{1}{39}$ \\
\hline
\end{tabular}

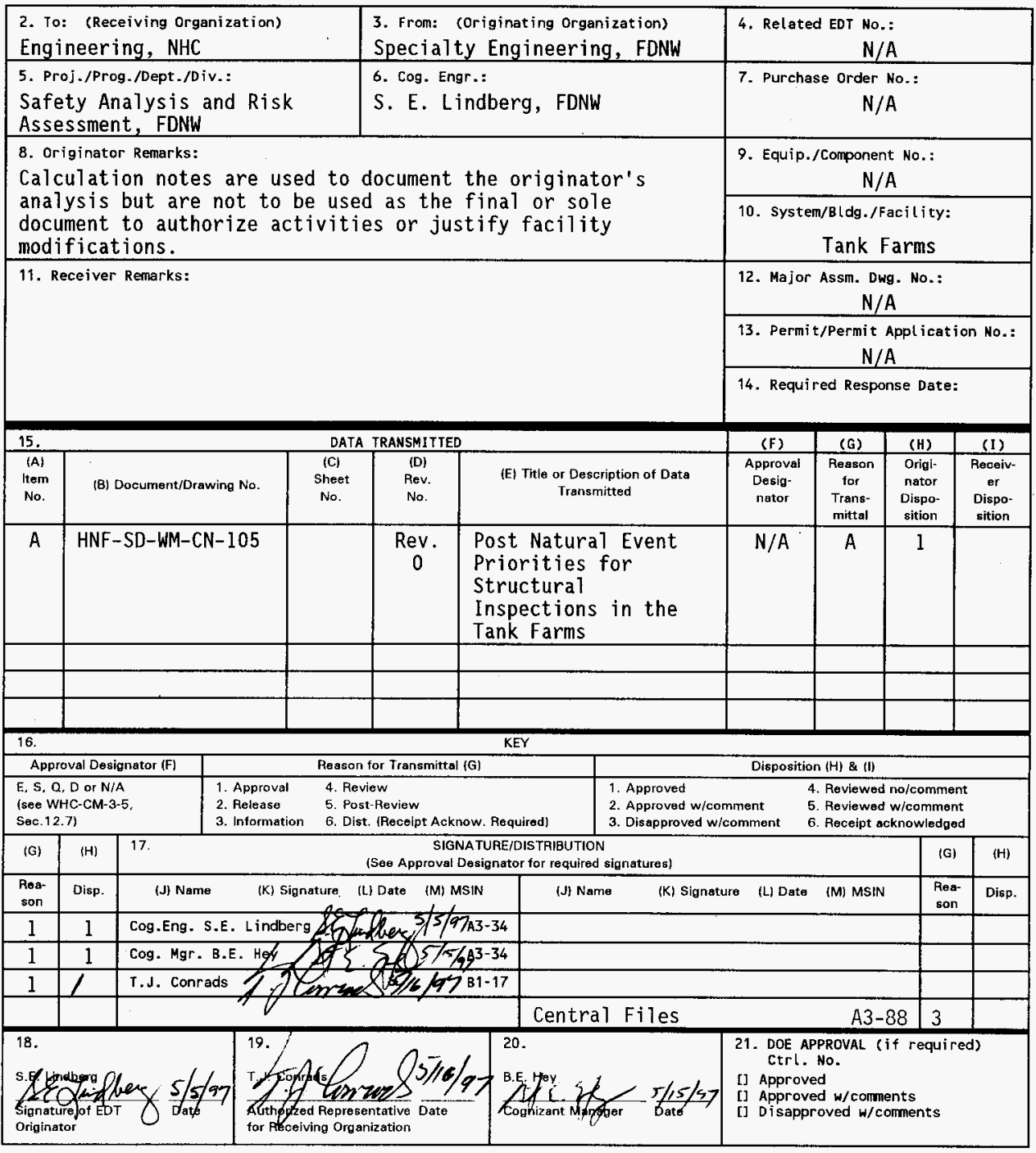

BD-7400-172-2 (04/94) GEF097 


\title{
Post Natural Phenomena Event Priorities for Structural Investigations in the Tank Farms
}

\author{
S. E. Lindberg \\ Fluor Daniel Northwest, Inc. \\ Richland, WA 99352 \\ U.S. Department of Energy Contract DE-AC06-96RL13200 \\ EDT/ECN : 621239 \\ Org Code: 403 \\ UC: 503 \\ Charge Code: P3HT02 \\ B\&R Code: YN0100000 \\ Total Pages: 32
}

Key Words: Natural phenomena, tank farms, seismic, earthquake, structural inspection

Abstract: Prioritized list for structural inspections in the tank farms following a severe natural phenomena event.

TRADEMARK DISCLAIMER. Reference herein to any specific comercial product, process, or service by trade name, trademark, manufacturer, or otherwise, does not necessarily constitute or imply its endorsement, recommendation, or favoring by the United States Government or any agency thereof or its contractors or subcontractors.

Printed in the United States of America. To obtain copies of this document, contact: Document Control Services, P.O. Box 950, Mailstop H6-08, Richland WA 99352, Phone (509) 372-2420; Fax $(509)$ 376-4989.
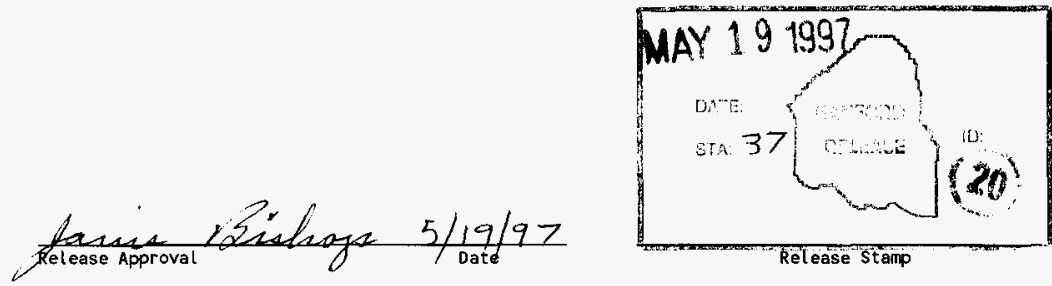

Approved for Public Release 
HNF-SD-WM-CN-105, Rev. 0

POST NATURAL PHENOMENA EVENT PRIORITIES

FOR STRUCTURAL INVESTIGATIONS IN THE TANK FARMS

S.E. Lindberg

Fluor Daniel Northwest, Inc.

Richl and, Washington

May, 1997 
HNF-SD-WM-CN-105, Rev. 0

\section{POST NATURAL PHENOMENA EVENT PRIORITIES \\ FOR STRUCTURAL INVESTIGATIONS IN THE TANK FARMS}

\subsection{Purpose}

The purpose of this task is to prioritize the tanks and tank farms having the greatest potential for structural damage following a significant natural phenomena. An event of sufficient magnitude could cause widespread structural damage throughout the tank farms. The damage caused by a seismic event may not be observable because many components such as transfer lines, tanks, or process pits are underground. Or, components over the waste tanks may be involved, with damage to ventilation system housings, or exhaust stacks. When requested to enter the tank farm facilities to inspect for structural damage due to natural phenomena, the investigating team needs to have previously considered the priorities of their actions so that they can complete their work in a timely fashion. Some aspects to be considered include the safety of the inspection team itself, the tank farm workers safety, environmental hazards, and the potential for hazardous materials affecting the public. The various tank farm facilities contain a significant amount of hazardous materials, both radiological and toxic.

This activity reviewed the current safety envelope of all of the waste tanks and determined a priority of inspection assuming a11 tanks were subject to a significant natura? phenomena.

\subsection{Scope}

The scope of this study includes all of the tank farm facilities within the farms themselves. There are 177 waste tanks in 18 tank farms. The physical scope of this study is limited to:

- $\quad$ Single-shell tanks (SSTs)

- Double-shell tanks (DSTs)

- Catch tanks in the tank farms

- Miscellaneous Underground Storage Tanks (MUSTs) in the tank farms

- Waste transfer systems in the tank farms.

\subsection{Prioritization Methodology}

To develop a prioritized structural inspection list, several steps are followed. The potential damage to the waste tank facilities in response to severe natural phenomena was reviewed, and priorities for inspection are proposed based on safety of the facility workers, on-site workers, and the public. A list of individual tanks and their characteristics such as whether or not the tank is included on a watchlist or if the tank is a known leaker, has been included. The waste tank histories were reviewed as well as the waste inventories; these are also incorporated in the list. An analysis of the 177 tanks was performed, and the attributes were ranked in order of importance to the safety functions of the tank farm facilities. The potential effects of years of high level waste storage on the tank structures were also considered. The various rankings are summed, and a prioritized list of individual tanks was developed to be used by the structural inspection team. 
A prioritized 1 ist of the 18 tank farms is also developed from the 1 ist of individual tank priorities.

\subsection{Assumptions}

The following assumptions are used in the analysis:

1. The study covers natural phenomena events that have the potential to affect all tank farms. Other types of events may only damage one or two farms, these events have not been included because they do not need to be prioritized.

2. The greater the inventory of high level waste in a tank, the greater the consequences of a gas release event (GRE) and deflagration following a severe seismic event.

3. Interim stabilized SSTs have less than 5000 gallons of pumpable liquid remaining in them, therefore the potential for a large spill from a ruptured interim stabilized tank is minimized.

4. If a waste tank normally has an operating active ventilation system, the assumption is that the ventilation system is needed to maintain cooling and confinement, so the loss of the ventilation system increases the ranking of its associated tank.

\subsection{Assessment of Natura1 Phenomena}

The Tank Waste Remediation System (TWRS) Basis for Interim Operation/Final Safety Analysis Report (BIO/FSAR) Natural Phenomena analysis identified high winds, lightning, and seismic events as potential significant hazards. For this analysis the lightning scenario has not been studied further because, though lightning strikes may cause a loss of power, or start fires, these are not scenarios that would be of concern for an area-wide structural inspection.

High winds also are not a significant concern for this study. There is a Standing Order (\#97-04) in the tank farms which instructs the operations staff in precautions for high wind conditions. Historically the tank farms have safely withstood sustained winds in excess of $128.75 \mathrm{~km} / \mathrm{h}(80 \mathrm{mi} / \mathrm{h})$. Because of this fact, the Emergency Response Guide for high winds in the tank farms has been deleted.

Another natural phenomena that was considered in the BI0/FSAR but its consequences were not found to be significant, is volcanic eruption and ashfall. The major detrimental effects of the ashfall are the abrasion of rotating machinery and ash buildup on inlet filters. These would not be the subject of a structural investigation following the volcanic eruption. Another concern with ashfall is the weight of the ash if a large amount is deposited on top of the waste tanks. A six inch layer of dry ash weighs about 25 pounds per square foot. It is capable of absorbing up to 10 pounds per square foot of water, for a total of 35 pounds per square foot. This weight has the potential of causing building roof distress, but would not cause a failure of the tank domes.

The case of a seismic event is the main concern for potential structural damage in the tank farms. An earthquake could cause several types of damage 
throughout the farms. A seismic event could damage many components in the various farms simultaneously. Potentially, the damage could result in:

- spray leaks in jumper pits or pump pits if a waste transfer is in progress and the jumper connections are damaged,

- the earthquake could cause the mixing or rollover of tank waste which could release entrapped flammable gases into the tank dome, a GRE. If the release caused a build-up of flammable gases beyond the lower flammability limit (LFL) a spark that is also generated by the seismic activity would result in a deflagration in the waste tank. Even if the GRE did not exceed the LFL in the tank dome, precautions need to be taken following a significant seismic event to prevent spark sources in potential pockets of high flammable gas.

- An underground high level waste tank could crack and allow a subsurface waste leak.

- Ground level components such as ventilation systems exhaust fan and filter housings, or exhaust system stacks could be damaged or toppled during a seismic event of sufficient magnitude.

\subsection{Inspection Priorities}

After a significant seismic event there is a high probability of LFL amounts of flammable gases in many waste tank domes. Extreme caution needs to be taken to avoid injuring the inspectors, and to prevent any spark sources during the structural inspection until the flammable gas concentration is known. The possibility also exists that some tanks may have experienced a flammabie gas deflagration caused by the seismic event. The earthquake can result in a significant GRE as well as causing ignition sources in the tanks as components may have the potential to swing around and strike each other during the events. In this second case, the vent system HEPA filters may be damaged, and extreme care must be taken to protect those entering the tank farms to prevent inhaling hazardous materials that may have been blown out of the damaged filter path.

There are two primary functions of the high level waste tanks and their associated facilities in the tank farms. The hazardous waste must remain cool enough that it does not boil or cause a tank bump, and the hazardous waste must be confined inside controlled areas to prevent injuring the facility worker, onsite workers, and the public. A significant seismic event can cause the disruption of the cooling and confining functions of the tank farm facilities. The structural inspection team should first look for any damaged components whose function is to cool the tank contents or to confine the hazardous materials inside the tanks.

The first priority should be to maintain, or restart any cooling, and provide a cooling and filtration path for tank air to move, i.e., to open any blocked air pathways from normally actively ventilated tanks or passively ventilated tanks. The components of interest then would include:

- active ventilation systems, comprised of:

- fans

- ductwork, exhaust stacks 
- HEPA filters

- filter housings

- tank penetrations such as process pits and their associated coverblocks, used to supply air to the waste tank

- passive ventilation systems, comprised of:

- HEPA filters

- filter housings

- air supply housings

Another concern following a seismic event arises if a waste transfer was in progress when the earthquake occurred. The ground movement could potentially damage the underground transfer lines and the connectors and associated piping that run through the process pits. Damage to the underground lines could eventually cause a surface pool, but would most likely stay under the ground level surface. However, damage in the process pits if a coverblock was crushed to an extent that the process pit was exposed, could allow a spray leak to occur. The first priority for the operations personnel who are performing the transfer procedure is to stop the transfer by shutting off the transfer pumps.

One consequence of a substantial seismic event could be cracking or a large split in an underground waste tank. The DSTs were all designed and built to withstand a $0.25 \mathrm{~g}$ seismic event. A review of the criteria used in the design and construction of the SSTs determined that they will withstand a ground acceleration of $0.20 \mathrm{~g}$. The SSTs have only one wall as a containment barrier so the waste would be released underground. The DSTs have a secondary tank to contain any leakage from the primary tank, so both tanks would have to be damaged in order to release the waste to the soil. In either case the leaking waste would be covered by a minimum of 15-55 feet of packed soil. The damage would not be obvious from the ground level other than by level instrument readings or radiation probe instruments inserted in the ground. In general, the tanks most likely to be damaged, the SSTs, have the least amount of waste that could leak out of a damaged tank. Many of the SSTs have been interim stabilized to remove most of the liquid in the tank. In fact, there is only one of the 149 SSTs that has greater than 37,000 gallons of supernate (1iquid) waste. The solids in any SST are not likely to flow out to the soil even if the tank wall is cracked. So, though a split in the wall or bottom of a waste tank would be a costly and difficult problem to clean up, the safety implications for the worker or public are minimal.

All of the above concerns are considered in developing the prioritized list of tanks and tank farms.

It is assumed that the inspection team will have a radiation protection technician accompanying them to warn of hazardous dose rates and minimize exposures, as we 11 as a member of the tank farms operating staff that knows the normal components' functions and status of the farms and transfer systems.

The scope of this analysis includes all of the components located in the tank farms. Some smaller underground tanks are considered but not included in the priority lists because they are very small compared to the high level waste tanks, or they are only used during waste transfers. The small tanks could be damaged in a seismic event, but the consequences are extremely small compared to the large waste tanks. For the case of the tanks used in waste transfers, they also are small compared to the large waste tanks, and they 
would have a smaller chance of having a large GRE that could potentially cause a deflagration.

\subsection{Prioritized List for Structural Inspectors}

\subsection{Individual Tank Priorities}

To develop a priority list for structural inspections in the tank farms following a seismic event, many items are considered. These include the status of the waste tanks as watchlist tanks, the amount of waste in the tanks, whether they are SSTS or DSTs, if the SSTs have been interim stabilized, the age of the tanks, the types of waste stored in the tanks, and whether or not the tanks are actively or passively ventilated. The data are shown on Table A-1 in Appendix A.

The numbers in the second column, Vent System Rank, are assigned according to the normal ventilation status of the associated tank. If the tank normally has an active ventilation system for cooling and confinement, the tank was assigned a ' 5 '. If the tank normally uses a passive breathing ventilation system, and the tank is on the watchlist for flammable gas, it was assigned a ' 4 '. If the tank normally uses a passive breathing system and it is a watchlist tank but not for flammable gas, then it is assigned a zero (0). The rest of the tanks have a dash (-) listed in this column. The rankings of 5,4 , and 0 are chosen to indicate the importance of the operable ventilation systems and their association with the prevention of a buildup of flammable gas in the tank domes as well as the cooling function.

Column three (3) in Table A-1, Inventory Rank, accounts for the inventory of high level waste in the waste tank. The greater the amount of inventory in the waste tank, the greater the amount of flammable gas that can be generated, or the greater risk of the inventory of waste in the tanks turning over and releasing a large amount of trapped flammable gas. Also, the greater the inventory, the greater the amount of waste that could spill in the case of a split tank. The risk of spilling a large amount of waste is much greater if there is a large inventory to be spilled. The rankings of 4,2 , and 0 are based on the increasing risk with a larger inventory.

The inventory ranking is based on the following:

Rank $4=\geq 50 \%$ of the total capacity of the tank is filled

Rank $2=10-49.9 \%$ of the total capacity of the tank is filled

Rank $0=<10 \%$ of the tank is filled.

An ' $X$ ' in column four (4) indicates that the tank has been assigned to a watchlist. Those not assigned to a watchlist just have a dash (-) in this column. The assignment to a watchlist indicates that the tank has a higher probability of a deflagration or fire caused by a seismic event, or that the tank contains very high temperature waste that has potentially caused stress to the tank wall.

The fifth (5) column is for Tank leaks. The tank is assigned a value rank of four to account for being a known leaker. If the tank is a leaker, the integrity is already questionable, and a seismic event could potentially increase the area damaged. The ranking of four for this column is to show the 
importance of a tank with a split already in it and the risk of the crack increasing due to a seismic event.

The sixth (6) column accounts for those tanks that were allowed to Selfconcentrate. The tanks indicating 'boil' were allowed to boil as a means of cooling, this only includes SSTs. These tanks are assigned a value rank of three to account for the structural stress to the tank wall from the high temperatures experienced. The ranking of three assigned to the tanks that were allowed to boil indicates that the stresses on the tanks were extreme but that the risk of them being damaged in a seismic event is not as great as the tanks with a crack in them already (those tanks are assigned a rank of four).

The seventh ( 7 ) column, heat const, accounts for the waste constituents put into the tank that are significant in the amount of heat generated. A search was made of the historical data of the waste inventories of the tanks. Some waste is known to be hotter than most. A value ranking of one was added for each constituent known to have been added to a tank. The rankings indicate the importance of the waste constituents and their effect on the stress to the tank walls. Current tank waste temperatures are not chosen for this column because the heat has decreased over the years. The constituents added to the tanks over the years give an indication of the history of the stresses and potential degradation to the tank walls due to the internal waste temperature. Symbols include:

Aging: aging waste

B: B-Plant high level waste

P: PUREX high level waste

PNF: Partial neutralized waste

1C: First cycle waste

IWW: PUREX \#l acid concentrator waste

The eighth (8) column Interim Stabilization accounts for the fact that many SSTs have had the majority of the supernate and interstitial fluid pumped out of them. An 'IS' in this column indicates the tank has been interim stabilized. This process includes pumping most of the liquid out of the tank so that the tank has less than 50,000 gallons of drainable interstitial liquid and less than 5,000 gallons of supernate liquid. The less liquid in the tank, the less that can flow out if the tank wall is damaged. Interim stabilization is considered when totaling the tank ranking in column ten.

Column nine (9), Tank age, is a ranking to account for the age of the tank. The older the tank, the greater the probability of a seismic event causing structural damage. The higher ranking accounts for the increased risk of aging on the tank liners and walls. The rankings are as follows:

+2 - the tank was built in the 1940 s or 1950 s, it is $40-50$ years old,

+1 - the tank was built in the 1960 s or $1970 \mathrm{~s}$, it is $20-30$ years old, 0 - the tank was built since 1980 .

To calculate the total risk indicator shown in column ten (10), a sum was made of the factors, or rankings, assigned in columns 2-9. For the tanks that are assigned to a watchlist, this sum was multiplied by 1.25 to account for the higher risk of the waste in these tanks. The total risk factor for the tanks that have been interim stabilized has been multiplied by 0.5 to account for the lower risk of a tank with a minimum of liquid inventory. Interim stabilization removes the great majority of the liquid in a tank. The tank 
HNF-SD-WM-CN-105, Rev. 0

inventory is pumped down to $<5,000$ gallons of supernate and $<50,000$ gallons of drainable interstitial liquid. Dividing the total risk indicator by two (multiplying by 0.5 ) shows the importance of the decreased risk due to the pumping of the liquid out of the tank. The factors in this column are in the range of 1 (the lowest risk) to 22.5 (the highest risk). This risk indicator was then used to rank the individual tanks in Table 7-1.

The following four columns are for information concerning the potential and actual inventory in each of the tanks. Column eleven (11) is the full tank volume capacity of each waste tank, column twelve (12) is the actual total inventory of the tank. Columns thirteen and fourteen show the amount of liquids and solids in each of the waste tanks. A-1:

The following table is a 1 ist of the tanks and their rankings from Table

Table 7-1. Tank Rankings

\begin{tabular}{|c|l||}
\hline Rank Tota1 & Waste Tank \\
\hline 22 & SX-104 \\
\hline 17 & $\mathrm{~A}-101, \mathrm{AX}-101, \mathrm{SX}-102, \mathrm{SX}-105$ \\
\hline 15 & $\mathrm{SX}-101, \mathrm{SX}-106, \mathrm{SY}-101$ \\
\hline 14 & $\mathrm{AY}-101$ \\
\hline 13 & $\mathrm{~S}-111, \mathrm{~S}-112, \mathrm{SX}-103, \mathrm{U}-103, \mathrm{U}-109, \mathrm{SY}-103$ \\
\hline 12 & $\begin{array}{l}\text { S-102, T-110, T-111, U-105, U-107, U-108, AW-101, AY-102, AZ- } \\
101, \mathrm{AZ}-102, \mathrm{SY}-102\end{array}$ \\
\hline 11 & $\mathrm{BY}-106, \mathrm{C}-106, \mathrm{~S}-101, \mathrm{AN}-103, \mathrm{AN}-104, \mathrm{AN}-105$ \\
\hline 10 & $\mathrm{BY}-105, \mathrm{U}-111, \mathrm{AW}-104$ \\
\hline 9 & $\mathrm{SX}-109, \mathrm{AN}-102, \mathrm{AN}-107, \mathrm{AP}-101, \mathrm{AP}-102, \mathrm{AP}-105$ \\
\hline 8 & $\mathrm{AW}-103, \mathrm{AW}-105, \mathrm{AW}-106$ \\
\hline 7 & $\mathrm{~A}-104, \mathrm{~A}-105, \mathrm{AX}-103, \mathrm{C}-103, \mathrm{~S}-106, \mathrm{~S}-109, \mathrm{~T}-104, \mathrm{AN}-101, \mathrm{AP}-$ \\
\hline 106
\end{tabular}




\begin{tabular}{||l|l||}
\hline 3 & $\begin{array}{l}\mathrm{A}-102, \mathrm{~B}-104, \mathrm{~B}-202, \mathrm{BX}-101, \mathrm{BX}-107, \mathrm{BX}-108, \mathrm{BY}-101, \mathrm{BY}-104, \\
\mathrm{BY}-109, \mathrm{BY}-110, \mathrm{BY}-111, \mathrm{C}-102, \mathrm{C}-201, \mathrm{C}-202, \mathrm{C}-203, \mathrm{C}-204, \mathrm{~S}-\end{array}$ \\
& $\begin{array}{l}105, \mathrm{~S}-108, \mathrm{~S}-110, \mathrm{~T}-103, \mathrm{~T}-105, \mathrm{~T}-106, \mathrm{~T}-108, \mathrm{~T}-201, \mathrm{~T}-203, \\
\mathrm{~T}-204, \mathrm{TX}-101, \mathrm{TX}-106, \mathrm{TX}-107, \mathrm{TX}-112, \mathrm{TX}-118, \mathrm{TY}-106, \mathrm{U}-101, \\
\mathrm{U}-112\end{array}$ \\
\hline 2 & $\begin{array}{l}\mathrm{B}-106, \mathrm{~B}-108, \mathrm{~B}-109, \mathrm{BX}-103, \mathrm{BX}-104, \mathrm{BX}-109, \mathrm{BX}-112, \mathrm{BY}-102, \\
\mathrm{BY}-112, \mathrm{C}-105, \mathrm{C}-107, \mathrm{C}-108, \mathrm{C}-109, \mathrm{C}-112, \mathrm{~T}-12, \mathrm{~T}-202, \mathrm{TX}- \\
102, \mathrm{TX}-103, \mathrm{TX}-108, \mathrm{TX}-109, \mathrm{TX}-111, \mathrm{U}-201, \mathrm{U}-202\end{array}$ \\
\hline 1 & $\mathrm{~B}-102, \mathrm{BX}-105, \mathrm{BX}-106, \mathrm{~T}-102, \mathrm{TX}-104, \mathrm{TY}-102, \mathrm{U}-203, \mathrm{U}-204$ \\
\hline
\end{tabular}

\subsection{Tank Farm Priorities}

Table A-1 includes the structural inspection priority ranking for each of the 177 high level waste tanks following a natural phenomena event. The individual rankings are important to determine which tanks have the highest risk in case of a loss of cooling or confinement of its hazardous waste inventory. However, it may not be practical for the inspection team to be entering and leaving several tank farms in order to inspect the high priority tanks first. The tank farms then, have also been ranked and listed in Table B-l, according to the average tank risk priorities discovered in this analysis. This list was determined by summing the total risk indicators from Table A-l for all of the tanks in each tank farm, and dividing by the number of tanks in the farm, to give an average risk indicator for the farm.

\subsection{References}

1. PNNL-11391, 1996, Gas Retention and Release Behavior in Hanford SingleShell Waste Tanks, Pacific Northwest National Laboratory, Richland, Washington.

2. WHC-EP-0182-102, 1996, Waste Tank Summary Report for Month Ending September 30, 1996, Westinghouse Hanford Company, Richland, Washington.

3. WHC-EP-0182-103, 1996, Waste Tank Summary Report for Month Ending October 31, 1996, Westinghouse Hanford Company, Richland, Washington.

4. WHC-IP-0839-TF, Rev. 0, 1996, Tank Farms Emergency Response Guides, Guide No. I, "Volcanic Eruption and Ashfall", Westinghouse Hanford Company, Richland, Washington.

5. WHC-IP-0839-TF, Rev. 0, 1996, Tank Farms Emergency Response Guides, Guide No. J, "Seismic Event", Westinghouse Hanford Company, Richland, Washington.

6. WHC-SD-TWR-RPT-002, Rev. 0, 1996, Structural Integrity and Potential Failure Modes of the Hanford High-Level Waste Tanks, Westinghouse Hanford Company, Richland, Washington.

7. WHC-SD-TWR-RPT-003, Rev. 0, 1996, DELPHI Expert Panel Evaluation of Hanford High Level Waste Tank Failure Modes and Release Quantities, Westinghouse Hanford Company, Richland, Washington. 
HNF-SD-WM-CN-105, Rev. 0

8. WHC-SD-W236A-TI-002, Rev. 1, 1996, Probabilistic Seismic Hazard Analysis, DOE Hanford Site, Washington, Westinghouse Hanford Company, Richland, Washington.

9. WHC-SD-WM-TI-764, Rev. 0, 1996, Hazard Analysis Database Report, Westinghouse Hanford Company, Richland, Washington.

10. WHC-SD-WM-TI-773, Rev. 0, 1996, Hazards Analysis Results Report, Westinghouse Hanford Company, Richland, Washington. 
HNF-SD-WM-CN-105, Rev. 0

\section{APPENDIX A}

List of Priorities for Structural Inspections 
HNF-SD-WM-CN-105, Rev. 0

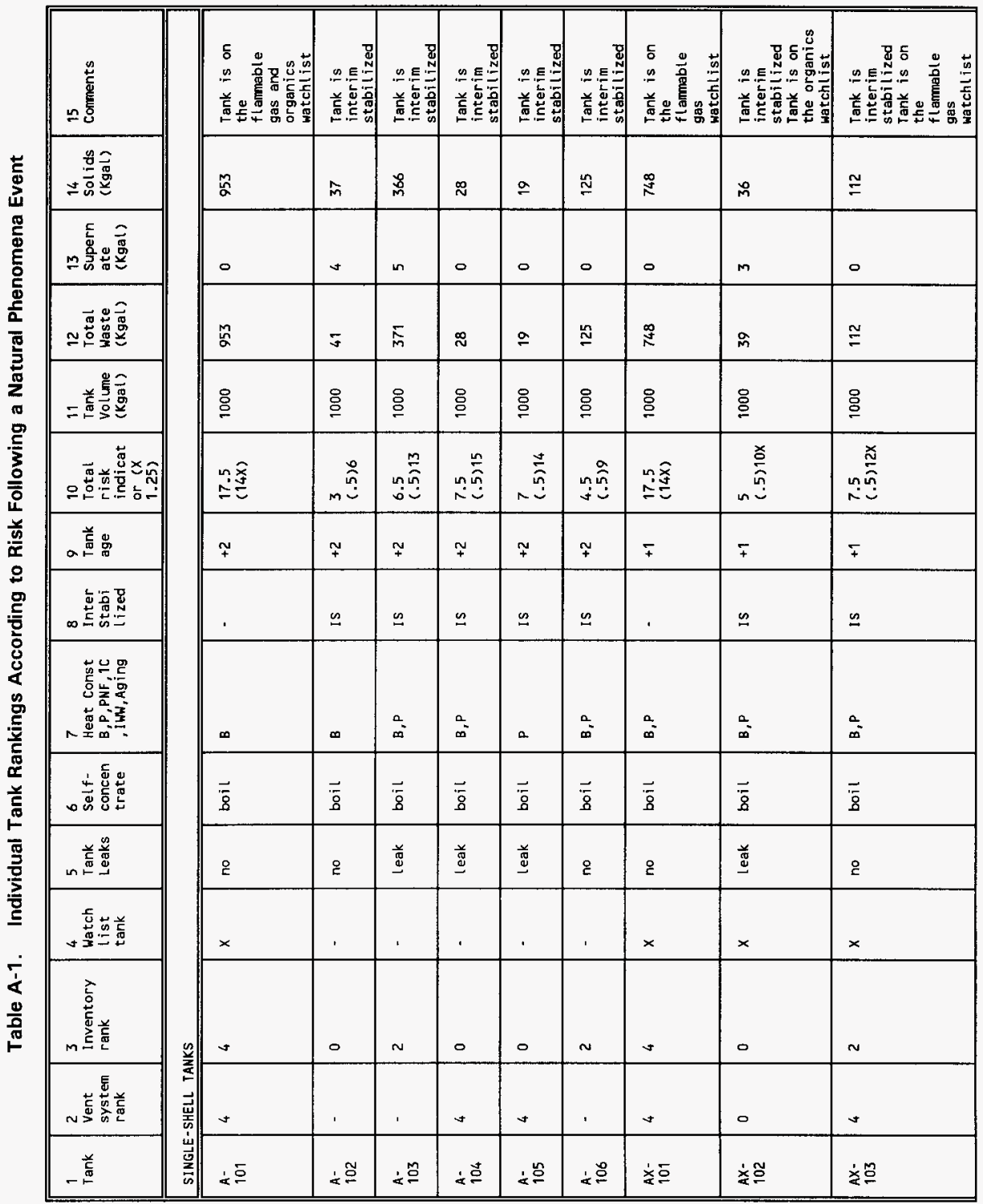


HNF-SD-WM-CN-105, Rev. 0

\begin{tabular}{|c|c|c|c|c|c|c|c|c|c|c|c|c|c|}
\hline 营 & 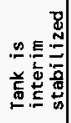 & 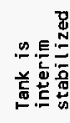 & 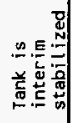 & 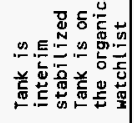 & 点 & 焉 & 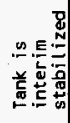 & 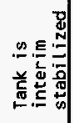 & 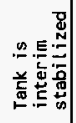 & 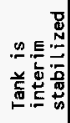 & 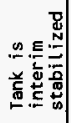 & 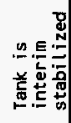 & 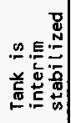 \\
\hline 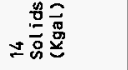 & n & $\stackrel{m}{=}$ & జ & in & 몸 & 品 & $\cong$ & 克 & $d$ & $\widehat{\underline{M}}$ & ב & 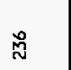 & m \\
\hline 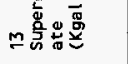 & 0 & 0 & $\checkmark$ & 0 & - & 0 & - & - & 0 & 0 & - & - & $\mathrm{m}$ \\
\hline 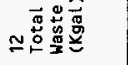 & $n$ & $\stackrel{m}{=}$ & m & in & 点 & 品 & $\Xi$ & $\stackrel{\curvearrowleft}{\mathscr{0}}$ & ¿ & $\cong$ & $\stackrel{0}{\sim}$ & ฒิ & $\tilde{m}$ \\
\hline$=$ & ฏ̊ & 品 & 品 & ถి & 品 & 品 & ถึ & ถి & n్ & 品 & ஜึ & 只 & 品 \\
\hline 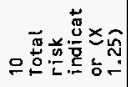 & 约 & 设 & $\stackrel{\widetilde{n}}{\tilde{n}}$ & 的 & ma & 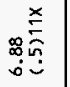 & กุ & 设 & กู่ & ๙ู่ & 胫 & ํํำ & $\stackrel{\infty}{\widehat{n}}$ \\
\hline 盖尊 & $\mp$ & 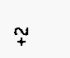 & $\simeq$ & 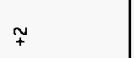 & ¥ & $\simeq$ & 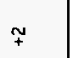 & $\simeq$ & $\approx$ & 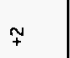 & 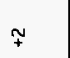 & $¥$ & 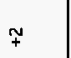 \\
\hline 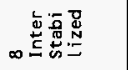 & 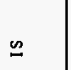 & 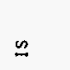 & $\cong$ & $\cong$ & $\cong$ & $\cong$ & $\cong$ & $\cong$ & $\cong$ & $\cong$ & $\stackrel{\Omega}{2}$ & $\cong$ & $\cong$ \\
\hline 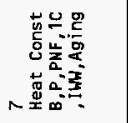 & $\stackrel{2}{\infty}$ & $\infty$ & , & . & $\cong$ & $\cong$ & $\cong$ & 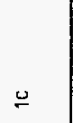 & 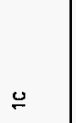 & $\cong$ & $\begin{array}{l}\vdots \\
\vdots \\
a^{\circ}\end{array}$ & $\cong$ & $\stackrel{\vdots}{\circ}$ \\
\hline 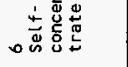 & $\overline{8}$ & ๕ & , & . & & & & & . & . & , & & \\
\hline 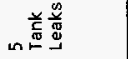 & 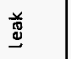 & 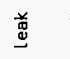 & \& & 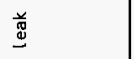 & \& & 鸹 & $\stackrel{\circ}{\circ}$ & 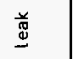 & $\stackrel{9}{9}$ & \& & 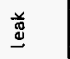 & 咆 & 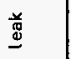 \\
\hline 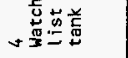 & & & ' & $\times$ & & & & & & . & ' & & \\
\hline 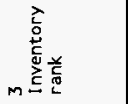 & 0 & $\sim$ & 0 & $N$ & $\checkmark$ & $\checkmark$ & $\sim$ & $\sim$ & $N$ & N & $\sim$ & $N$ & 0 \\
\hline 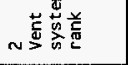 & & & ' & 0 & & & & & & . & . & & \\
\hline - & 紊泀 & io & $\dot{\omega} \tilde{\tilde{O}}$ & $\dot{m} \stackrel{m}{\circ}$ & 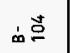 & i. & m. & $\therefore$ is & i & 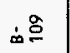 & 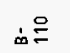 & in $E$ & in $\stackrel{\simeq}{\simeq}$ \\
\hline
\end{tabular}




\begin{tabular}{|c|c|c|c|c|c|c|c|c|c|c|c|c|c|c|}
\hline 1 Tank & $\begin{array}{l}2 \\
\text { Vent } \\
\text { system } \\
\text { rank }\end{array}$ & $\begin{array}{l}3 \\
\text { Inventory } \\
\text { rank }\end{array}$ & $\begin{array}{l}4 \\
\text { Watch } \\
\text { list } \\
\text { tank }\end{array}$ & $\begin{array}{l}5 \\
\text { Tank } \\
\text { Leaks }\end{array}$ & $\begin{array}{l}6 \\
\text { Self- } \\
\text { concen } \\
\text { trate }\end{array}$ & $\begin{array}{l}7 \\
\text { Heat Const } \\
\text { B,P,PNF, C } \\
\text {,I I W Aging }\end{array}$ & $\begin{array}{l}8 \\
\text { Inter } \\
\text { Stabi } \\
\text { lized }\end{array}$ & $\begin{array}{l}9 \\
\text { Tank } \\
\text { age }\end{array}$ & $\begin{array}{l}10 \\
\text { Total } \\
\text { risk } \\
\text { indicat } \\
\text { or }(x \\
1.25) \\
\end{array}$ & $\begin{array}{l}11 \\
\text { Tank } \\
\text { Volume } \\
\text { (Kgal) }\end{array}$ & $\begin{array}{l}12 \\
\text { Total } \\
\text { Waste } \\
\text { (Kgal) }\end{array}$ & $\begin{array}{l}13 \\
\text { Supern } \\
\text { ate } \\
\text { (Kgal) }\end{array}$ & $\begin{array}{l}14 \\
\text { Sol ids } \\
\text { (Kgal) }\end{array}$ & $\begin{array}{l}15 \\
\text { Comments }\end{array}$ \\
\hline $\begin{array}{l}8-- \\
201\end{array}$ & & 4 & - & leak & - & - & IS & +2 & $\begin{array}{l}5 \\
(.5) 10\end{array}$ & 55 & 29 & 1 & 28 & $\begin{array}{l}\text { Tank is } \\
\text { interim } \\
\text { stabilized }\end{array}$ \\
\hline $\begin{array}{l}\text { B- } \\
202\end{array}$ & - & 4 & - & no & $\cdot$ & - & Is & +2 & $\begin{array}{l}3 \\
6.5) 6\end{array}$ & 55 & 27 & 0 & 27 & $\begin{array}{l}\text { Tank is } \\
\text { interim } \\
\text { stabilized }\end{array}$ \\
\hline $\begin{array}{l}\text { B- } \\
203\end{array}$ & - & 4 & & leak & - & - & Is & +2 & $\frac{5}{6.5) 10}$ & 55 & 51 & 1 & 50 & $\begin{array}{l}\text { Tank is } \\
\text { interim } \\
\text { stabilized }\end{array}$ \\
\hline $\begin{array}{l}\text { B- } \\
204\end{array}$ & $\cdot$ & 4 & $\cdot$ & leak & - & - & Is & +2 & $\begin{array}{l}5 \\
(.5) 10\end{array}$ & 55 & 50 & 1 & 49 & $\begin{array}{l}\text { Tank is } \\
\text { inter im } \\
\text { stabilized }\end{array}$ \\
\hline $\begin{array}{l}\text { BX- } \\
101\end{array}$ & - & 0 & $\cdot$ & leak & . & - & Is & +2 & $3.5) 6$ & 530 & 43 & 1 & 42 & $\begin{array}{l}\text { Tank is } \\
\text { interim } \\
\text { stabilized }\end{array}$ \\
\hline $\begin{array}{l}\mathrm{BX}- \\
102\end{array}$ & - & 2 & - & leak & - & - & is & +2 & $4.5) 8$ & 530 & 96 & 0 & 96 & $\begin{array}{l}\text { Tank is } \\
\text { inter im } \\
\text { stabilized }\end{array}$ \\
\hline $\begin{array}{l}\text { BX- } \\
103\end{array}$ & - & 2 & - & no & - & $P$ & is & +2 & $\begin{array}{l}2.5 \\
(.5) 5\end{array}$ & 530 & 68 & 6 & 62 & $\begin{array}{l}\text { Tank is } \\
\text { interim } \\
\text { stabilized }\end{array}$ \\
\hline $\begin{array}{l}\mathrm{BX}- \\
104\end{array}$ & - & 2 & - & no & - & - & is & +2 & $\begin{array}{l}2 \\
6.5) 4\end{array}$ & 530 & 99 & 3 & 96 & $\begin{array}{l}\text { Tank is } \\
\text { inter im } \\
\text { stabilized }\end{array}$ \\
\hline $\begin{array}{l}\text { BX- } \\
105\end{array}$ & - & 0 & - & no & - & - & is & +2 & $\begin{array}{l}1 \\
(.5) 2\end{array}$ & 530 & 51 & 5 & 46 & $\begin{array}{l}\text { Tank is } \\
\text { interim } \\
\text { stabilized }\end{array}$ \\
\hline $\begin{array}{l}\text { BX- } \\
106\end{array}$ & - & 0 & - & no & - & - & Is & +2 & $\begin{array}{l}1 \\
(.5) 2\end{array}$ & 530 & 38 & 0 & 38 & $\begin{array}{l}\text { Tank is } \\
\text { interim } \\
\text { stabilized }\end{array}$ \\
\hline $\begin{array}{l}\text { BX- } \\
107\end{array}$ & - & 4 & - & no & - & IC & is & +2 & $\begin{array}{l}3.5 \\
(.5) 7\end{array}$ & 530 & 345 & 1 & 344 & $\begin{array}{l}\text { Tank is } \\
\text { interim } \\
\text { stabilized }\end{array}$ \\
\hline $\begin{array}{l}\text { BX- } \\
108\end{array}$ & $\cdot$ & 0 & $\cdot$ & leak & - & - & is & +2 & $\begin{array}{l}3 \\
(.5) 6\end{array}$ & 530 & 26 & 0 & 26 & $\begin{array}{l}\text { Tank is } \\
\text { interim } \\
\text { stabilized }\end{array}$ \\
\hline $\begin{array}{l}\text { BX- } \\
109\end{array}$ & - & 2 & - & no & - & IC & Is & +2 & $\begin{array}{l}2.5 \\
6.5) 5\end{array}$ & 530 & 193 & 0 & 193 & $\begin{array}{l}\text { Tank is } \\
\text { inter im } \\
\text { stabilized }\end{array}$ \\
\hline $\begin{array}{l}\text { BX- } \\
110\end{array}$ & - & 2 & & leak & - & IC & is & +2 & $\begin{array}{l}4.5 \\
(.5) 9\end{array}$ & 530 & 207 & 3 & 204 & $\begin{array}{l}\text { Tank is } \\
\text { inter im } \\
\text { stabilized }\end{array}$ \\
\hline
\end{tabular}




\begin{tabular}{|c|c|c|c|c|c|c|c|c|c|c|c|c|c|c|c|}
\hline 总 & 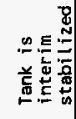 & 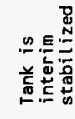 & 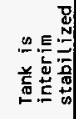 & 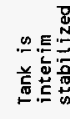 & & 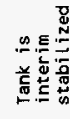 & & & 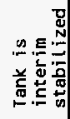 & 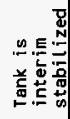 & & 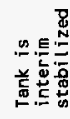 & 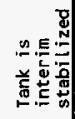 & 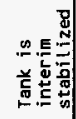 & 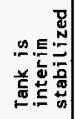 \\
\hline 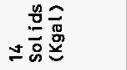 & $\Phi$ & 古 & 命 & ลิ & : & 号 & 总 & ีี & : & $\stackrel{\widetilde{N}}{\mathbb{N}}$ & $\underset{\mathfrak{G}}{\widetilde{N}}$ & $\stackrel{\infty}{\circ}$ & $\underset{g}{g}$ & న్ & 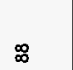 \\
\hline 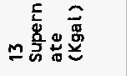 & - & - & 0 & 0 & 0 & 0 & 0 & 0 & 0 & 0 & 0 & 0 & 0 & 0 & 0 \\
\hline 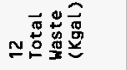 & ๕ & $\stackrel{n}{\circ}$ & 吣 & ล & : & 号 & m & 吉 & 足 & జึ & $\underset{\widetilde{G}}{\mathbb{7}}$ & $\stackrel{\infty}{\circ}$ & 学 & $\overline{\text { d }}$ & ஐ \\
\hline$=$ & 品 & 甪 & 吕 & 婴 & $\stackrel{\infty}{\mathfrak{n}}$ & $\stackrel{\infty}{\stackrel{\infty}{\kappa}}$ & $\stackrel{\infty}{\kappa}$ & $\stackrel{\infty}{\kappa}$ & 変 & 吕 & $\stackrel{\infty}{\stackrel{\infty}{n}}$ & $\stackrel{\infty}{\kappa}$ & 咒 & 㸃 & 兽 \\
\hline 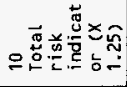 & 番 & กับ & m & N & 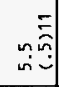 & m & 웅 & $=$ & 该 & $\stackrel{\infty}{\widehat{\overbrace{}}}$ & นn & min & mi & น & นุ่ \\
\hline 慈 & $\approx$ & $\approx$ & $\approx$ & $\approx$ & $\mp$ & $\mp$ & $\mp$ & $\mp$ & $\approx$ & $\approx$ & $\mp$ & $\mp$ & 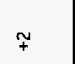 & $\simeq$ & $\approx$ \\
\hline 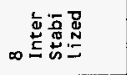 & $\cong$ & 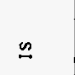 & $\cong$ & $\simeq$ & $\simeq$ & $\underline{\sim}$ & . & , & 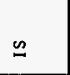 & 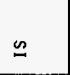 & & $\cong$ & $\cong$ & 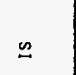 & $\approx$ \\
\hline 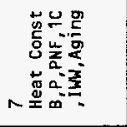 & $\stackrel{\varphi}{\circ}$ & 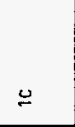 & & . & a & & , & $\cong$ & $\underline{\square}$ & , & 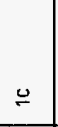 & 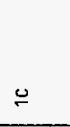 & ' & . & a \\
\hline 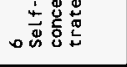 & I & . & . & . & . & & ' & ' & ' & ' & & . & & & . \\
\hline 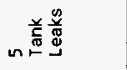 & 总 & g & உ & 울 & $\stackrel{\text {. }}{\underline{\Xi}}$ & $\stackrel{\circ}{\circ}$ & 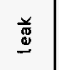 & $\stackrel{\text { g }}{\mathrm{g}}$ & 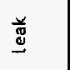 & 总 & $\stackrel{\circ}{\complement}$ & ㅇ & ㅇ & $\stackrel{2}{\varepsilon}$ & 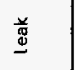 \\
\hline 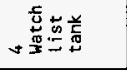 & . & . & & . & . & & & & , & & & . & & & \\
\hline 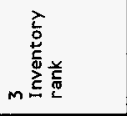 & $\sim$ & $\sim$ & s & $\sim$ & 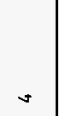 & $a$ & $\checkmark$ & $\checkmark$ & rv & $n$ & $\checkmark$ & v & $\checkmark$ & $\sim$ & $\sim$ \\
\hline 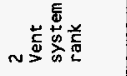 & . & . & & . & 0 & & 0 & 0 & . & & 0 & ' & & & . \\
\hline 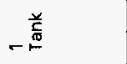 & $\dot{x} \equiv$ & $\dot{x} \cong$ & $\frac{1}{\mathbf{m}} \overline{0}$ & 亩芩 & 产耐 & 言す & 容ㅇำ & 商品 & 产全 & 啇음 & 商总 & 商음 & 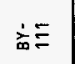 & $\frac{1}{\dot{m}} \cong$ & ن⿳亠口冋 \\
\hline
\end{tabular}




\begin{tabular}{|c|c|c|c|c|c|c|c|c|c|c|c|c|c|c|}
\hline $\begin{array}{l}1 \\
\text { Tank }\end{array}$ & $\begin{array}{l}2 \\
\text { Vent } \\
\text { system } \\
\text { rank }\end{array}$ & $\begin{array}{l}3 \\
\text { Inventory } \\
\text { rank }\end{array}$ & $\begin{array}{l}\text { Watch } \\
\text { list } \\
\text { tank }\end{array}$ & $\begin{array}{l}5 \\
\text { Tank } \\
\text { Leaks }\end{array}$ & $\begin{array}{l}6 \\
\text { Self- } \\
\text { concen } \\
\text { trate }\end{array}$ & $\begin{array}{l}7 \\
\text { Heat Const } \\
\text { B,P,PNF, } 1 \mathrm{C} \\
\text { IWW, Aging }\end{array}$ & $\begin{array}{l}8 \\
\text { Inter } \\
\text { Stabi } \\
\text { lized }\end{array}$ & $\begin{array}{l}9 \\
\text { Tank } \\
\text { age }\end{array}$ & $\begin{array}{l}10 \\
\text { Total } \\
\text { risk } \\
\text { indicat } \\
\text { or ( } x \\
1.25) \\
\end{array}$ & $\begin{array}{l}11 \\
\text { Tank } \\
\text { Volume } \\
\text { (Kgal) }\end{array}$ & $\begin{array}{l}12 \\
\text { Total } \\
\text { Waste } \\
\text { (Kgal) }\end{array}$ & $\begin{array}{l}13 \\
\text { Supern } \\
\text { ate } \\
\text { (Kgal) }\end{array}$ & $\begin{array}{l}14 \\
\text { Sol ids } \\
\text { (Kgal) }\end{array}$ & $\begin{array}{l}15 \\
\text { Comments }\end{array}$ \\
\hline $\begin{array}{l}c- \\
102\end{array}$ & - & 4 & $x$ & no & . & - & Is & +2 & $\begin{array}{l}3.75 \\
\text { (.5) } 6 x\end{array}$ & 530 & 316 & 0 & 316 & $\begin{array}{l}\text { Tank is } \\
\text { inter im } \\
\text { stabil ized } \\
\text { Tank is on } \\
\text { the organics } \\
\text { watchl ist }\end{array}$ \\
\hline $\begin{array}{l}\text { c- } \\
103\end{array}$ & 0 & 2 & $x$ & no & - & $B, P$ & - & +2 & $\begin{array}{l}7.5 \\
6 x\end{array}$ & 530 & 195 & 133 & 62 & $\begin{array}{l}\text { Tank is on } \\
\text { the organics } \\
\text { watchl ist }\end{array}$ \\
\hline $\begin{array}{l}c- \\
104\end{array}$ & - & 4 & - & no & - & $B, P, I W W$ & IS & +2 & $\begin{array}{l}4.5 \\
(.5) 9\end{array}$ & 530 & 295 & 0 & 295 & $\begin{array}{l}\text { Tank is } \\
\text { interim } \\
\text { stabilized }\end{array}$ \\
\hline $\begin{array}{l}\mathrm{c} . \\
105\end{array}$ & 0 & 2 & - & no & - & $P$ & Is & +2 & $\begin{array}{l}2.5 \\
(.5) 5 \\
\end{array}$ & 530 & 134 & 2 & 132 & \\
\hline $\begin{array}{l}c- \\
106\end{array}$ & 4 & 2 & $x$ & no & - & $P$ & - & +2 & $\begin{array}{l}11.3 \\
9 \mathrm{X}\end{array}$ & 530 & 229 & 32 & 197 & $\begin{array}{l}\text { Tank is on } \\
\text { the high } \\
\text { heat load } \\
\text { watchl ist }\end{array}$ \\
\hline $\begin{array}{l}\text { c- } \\
107\end{array}$ & $\cdot$ & 2 & - & no & - & $i c$ & IS & +2 & $\begin{array}{l}2.5 \\
(.5) 5\end{array}$ & 530 & 237 & 0 & 237 & $\begin{array}{l}\text { Tank is } \\
\text { interim } \\
\text { stabilized }\end{array}$ \\
\hline $\begin{array}{l}c- \\
108\end{array}$ & - & 2 & - & no & - & $1 \mathrm{c}$ & Is & +2 & $\begin{array}{l}2.5 \\
(.5) 5\end{array}$ & 530 & 66 & 0 & 66 & $\begin{array}{l}\text { Tank is } \\
\text { interim } \\
\text { stabilized }\end{array}$ \\
\hline $\begin{array}{l}c- \\
109\end{array}$ & - & 2 & - & no & - & $1 \mathrm{C}$ & is & +2 & $\begin{array}{l}2.5 \\
(.5) 5\end{array}$ & 530 & 66 & 4 & 62 & $\begin{array}{l}\text { Tank is } \\
\text { interim } \\
\text { stabilized }\end{array}$ \\
\hline $\begin{array}{l}c- \\
110\end{array}$ & - & 2 & - & leak & - & $1 \mathrm{C}$ & Is & +2 & $\begin{array}{l}4.5 \\
(.5) 9\end{array}$ & 530 & 178 & 1 & 177 & $\begin{array}{l}\text { Tank is } \\
\text { interim } \\
\text { stabilized }\end{array}$ \\
\hline $\begin{array}{l}c- \\
111\end{array}$ & - & 2 & - & leak & - & IC & is & +2 & $\begin{array}{l}4.5 \\
(.5) 9\end{array}$ & 530 & 57 & 0 & 57 & $\begin{array}{l}\text { Tank is } \\
\text { interim } \\
\text { stabilized }\end{array}$ \\
\hline $\begin{array}{l}\mathrm{c}- \\
112\end{array}$ & & 2 & & no & - & Ic & Is & +2 & $\begin{array}{l}2.5 \\
(.5) 5\end{array}$ & 530 & 104 & 0 & 104 & $\begin{array}{l}\text { Tank is } \\
\text { interim } \\
\text { stabilized }\end{array}$ \\
\hline $\begin{array}{l}c- \\
201\end{array}$ & - & 0 & - & leak & - & - & Is & +2 & $3.5) 6$ & 55 & 2 & 0 & 2 & $\begin{array}{l}\text { Tank is } \\
\text { inter in } \\
\text { stabilized }\end{array}$ \\
\hline $\begin{array}{l}c- \\
202\end{array}$ & - & 0 & - & leak & - & - & Is & +2 & $\begin{array}{l}3 \\
(.5) 6\end{array}$ & 55 & 1 & 0 & 1 & $\begin{array}{l}\text { Tank is } \\
\text { interim } \\
\text { stabilized }\end{array}$ \\
\hline
\end{tabular}


HNF-SD-WM-CN-105, Rev. 0

\begin{tabular}{|c|c|c|c|c|c|c|c|c|c|c|c|c|c|}
\hline 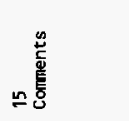 & 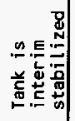 & 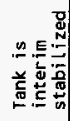 & & 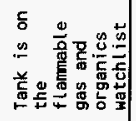 & & 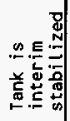 & 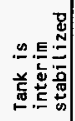 & & & & & & 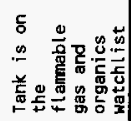 \\
\hline 土总总总 & in & $m$ & $\stackrel{n}{\sigma}$ & $\stackrel{a}{u}$ & $\bar{N}$ & $\stackrel{m}{\alpha}$ & 号 & $\underset{f}{\tilde{g}}$ & 饥 & ثี & : & 品 & 怘 \\
\hline M总喜高 & 0 & 0 & $\simeq$ & 0 & $\approx$ & - & 0 & $\checkmark$ & 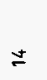 & 0 & 0 & 0 & 은 \\
\hline 焉嵒 & n & $m$ & 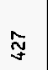 & 㤐 & $\underset{\sim}{\infty}$ & స్ & $\underset{7}{\stackrel{\circ}{4}}$ & $\stackrel{a}{\xi}$ & $\stackrel{0}{\stackrel{0}{m}}$ & క̊ & $\stackrel{\infty}{: 0}$ & 品 & ฉั \\
\hline 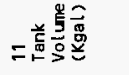 & นn & 崩 & $\stackrel{\infty}{\kappa}$ & $\stackrel{\infty}{\kappa}$ & 占 & 怘 & $\stackrel{\infty}{\kappa}$ & $\stackrel{\infty}{\kappa}$ & ח̊ & 梁 & $\stackrel{\infty}{\kappa}$ & 品 & $\stackrel{\infty}{n}$ \\
\hline 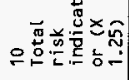 & $m \stackrel{\circ}{n}$ & m气 & $=$ & 무픔 & n & na & m气̃ & N & in & mุثิ & $n$ & นูบ & $\stackrel{\infty}{m} \stackrel{x}{=}$ \\
\hline م帝岸 & $\underset{+}{\sim}$ & $\stackrel{+}{+}$ & $\mp$ & 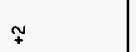 & $\simeq$ & $\mp$ & $\mp$ & $\mp$ & $\dddot{\sim}$ & 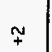 & $\dddot{\Psi}$ & $\underset{+}{\Psi}$ & $\underset{\leftarrow}{\mp}$ \\
\hline 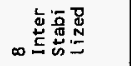 & $\cong$ & $\approx$ & . & ' & . & $\approx$ & $\stackrel{\infty}{\sim}$ & 1 & , & $\stackrel{\infty}{\sim}$ & 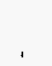 & $\approx$ & , \\
\hline 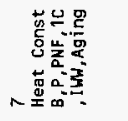 & . & . & $\begin{array}{l}\frac{\omega}{2} \\
\frac{0}{0}\end{array}$ & . & $\frac{u}{z}$ & $\frac{u}{2}$ & $\frac{u}{a}$ & 嵩 & $\frac{u}{a}$ & $\frac{u}{a}$ & 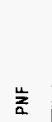 & $\frac{u}{a}$ & $\frac{u}{2}$ \\
\hline 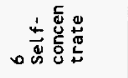 & ' & ' & $\overline{8}$ & ' & . & . & . & . & . & , & . & . & , \\
\hline 的总惫 & 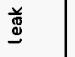 & 惫 & 을 & 울 & $\stackrel{2}{\complement}$ & 㴓 & $\stackrel{8}{8}$ & 을 & 옫 & 옫 & @ & $\stackrel{2}{g}$ & ̊ \\
\hline 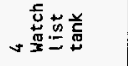 & . & ' & & $x$ & ' & . & , & . & ' & . & . & , & $x$ \\
\hline 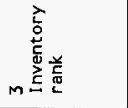 & 0 & 0 & + & $\checkmark$ & $N$ & N & $\checkmark$ & $\checkmark$ & $\sim$ & $\checkmark$ & $\checkmark$ & $\checkmark$ & $\checkmark$ \\
\hline 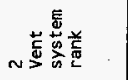 & . & ' & 0 & $\checkmark$ & 0 & . & , & 0 & 0 & 0 & 0 & 0 & ง \\
\hline 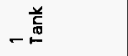 & ن & ن & is $\overline{0}$ & 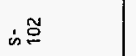 & is & is & is & $\therefore$ 의 & $\therefore$ is & $\therefore \stackrel{\infty}{\circ}$ & in 0 & in 은 & is $\equiv$ \\
\hline
\end{tabular}


HNF-SD-WM-CN-105, Rev. 0

\begin{tabular}{|c|c|c|c|c|c|c|c|c|c|}
\hline 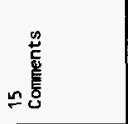 & 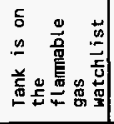 & 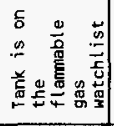 & 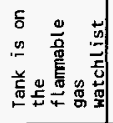 & 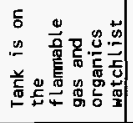 & 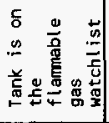 & 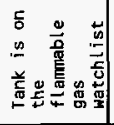 & 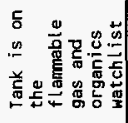 & 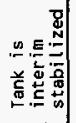 & 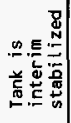 \\
\hline 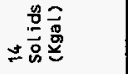 & N & 喿 & 筩 & กู้ & $\frac{\pi}{6}$ & 颔 & ח్ & $\Xi$ & $\widehat{\infty}$ \\
\hline 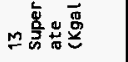 & 0 & - & 0 & - & 0 & 0 & $\overline{0}$ & 0 & 0 \\
\hline 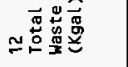 & $\tilde{\tilde{N}}$ & 号 & 急 & ถึ้ & $\frac{\pi}{0}$ & 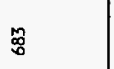 & ฒn & $\stackrel{t}{\circ}$ & $\hat{\infty}$ \\
\hline 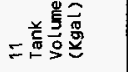 & 点 & 8 & $\stackrel{\circ}{\circ}$ & 음 & 음 & ஜ & 응 & 음 & ळ \\
\hline 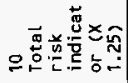 & $\stackrel{\infty}{m} \underset{=}{m}$ & 또 & $\stackrel{n}{=} \stackrel{x}{=}$ & $\stackrel{\infty}{m} \stackrel{x}{=}$ & $\stackrel{n}{\stackrel{n}{*} \stackrel{x}{\infty}}$ & $\stackrel{n}{=}$ & $\underline{\Perp} \stackrel{x}{\underline{x}}$ & $\underset{n}{n} \underset{\bar{n}}{\bar{n}}$ & นn \\
\hline 怠品 & $\dddot{+}$ & $\dddot{+}$ & $\stackrel{\sim}{+}$ & 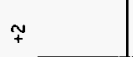 & 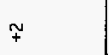 & $\widetilde{\leftarrow}$ & $\mp$ & $\widetilde{t}$ & $\simeq$ \\
\hline 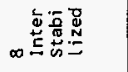 & . & . & . & ' & ' & ' & ' & $\stackrel{\sim}{\sim}$ & $\stackrel{2}{=}$ \\
\hline 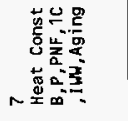 & 訔 & $\frac{u}{a}$ & $\frac{u}{\frac{1}{2}}$ & $\frac{\underline{z}}{\Delta}$ & $\frac{\frac{1}{z}}{a}$ & 产 & $\begin{array}{l}\frac{\omega}{2} \\
\frac{1}{2} \\
\infty^{-}\end{array}$ & . & \\
\hline 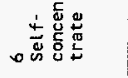 & ' & $\overline{8}$ & $\overline{8}$ & . & $\overline{8}$ & $\overline{8}$ & ' & $\overrightarrow{8}$ & $\overrightarrow{\overline{8}}$ \\
\hline 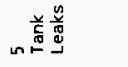 & ㅇ & $\stackrel{\circ}{\&}$ & $\stackrel{\circ}{ }$ & $\stackrel{\circ}{\Sigma}$ & 惫 & $\stackrel{9}{\check{1}}$ & ๕ & 总 & 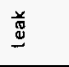 \\
\hline 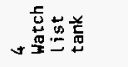 & $\times$ & $\times$ & $x$ & $\times$ & $\times$ & $\times$ & $\times$ & . & . \\
\hline m & + & $\sim$ & + & $\checkmark$ & + & $\Delta$ & $\checkmark$ & $\sim$ & 0 \\
\hline 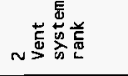 & + & $\checkmark$ & $\checkmark$ & + & $\checkmark$ & 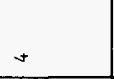 & $\checkmark$ & & . \\
\hline 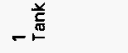 & is $\cong$ & 忞它 & 齐导 & 竦 & 产高 & 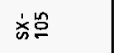 & 齐号 & 站 & 商号 \\
\hline
\end{tabular}


HNF-SD-WM-CN-105, Rev. 0

\begin{tabular}{|c|c|c|c|c|c|c|c|c|c|c|c|c|c|}
\hline 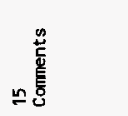 & 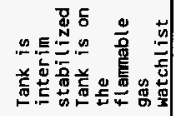 & 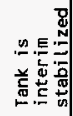 & 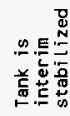 & 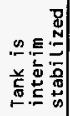 & 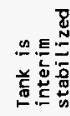 & 象 & 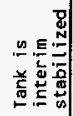 & 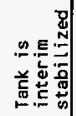 & 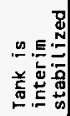 & 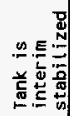 & & 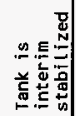 & 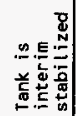 \\
\hline 을 & N & ๘ & $\stackrel{\text { น }}{ }$ & $\approx$ & 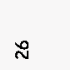 & $\Phi$ & $\simeq$ & E & $\underline{a}$ & N & 哭 & $\approx$ & $\stackrel{\circ}{ }$ \\
\hline 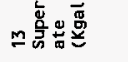 & 0 & 0 & 0 & 0 & 0 & 0 & 0 & - & $m$ & $\checkmark$ & 0 & 0 & $\sim$ \\
\hline 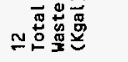 & 壳 & $\approx$ & $\cong$ & $\approx$ & $\stackrel{\sim}{N}$ & $\underline{\infty}$ & $\simeq$ & $\cong$ & $\approx$ & $\hat{\sim}$ & 总 & 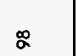 & $\bar{N}$ \\
\hline$=$ & 음 & 음 & 品 & 음 & $\stackrel{\circ}{\circ}$ & 음 & $\stackrel{\circ}{\circ}$ & 品 & ֻ̊ & (D) & 号 & हि & 品 \\
\hline 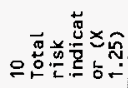 & 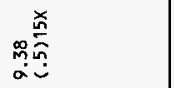 & 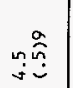 & 经 & นnู & นn & 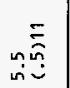 & 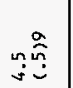 & น & $-\stackrel{\widetilde{n}}{3}$ & m & N & in & นกำ \\
\hline 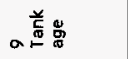 & 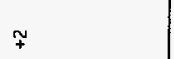 & $\mp$ & 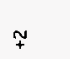 & $\mp$ & $\mp$ & $\uparrow$ & 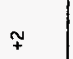 & ₹ & 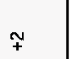 & ¥ & $\mp$ & $\approx$ & 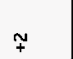 \\
\hline 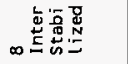 & $\cong$ & $\cong$ & $\cong$ & $\cong$ & 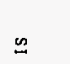 & 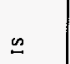 & $\approx$ & $\cong$ & $\cong$ & $\simeq$ & . & $\Leftrightarrow$ & $\cong$ \\
\hline 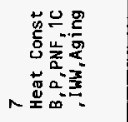 & . & & . & , & , & . & , & 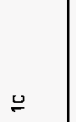 & & , & $\cong$ & $\cong$ & $\cong$ \\
\hline 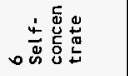 & $\overline{8}$ & $\overrightarrow{\bar{g}}$ & $\overline{\overline{8}}$ & $\overline{8}$ & $\overline{8}$ & $\overrightarrow{\overline{8}}$ & $\overline{8}$ & . & . & . & , & . & \\
\hline 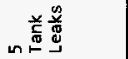 & 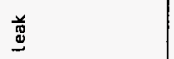 & 鸹 & 駡 & 递 & 菢 & 总 & 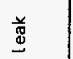 & 鸢 & ஜ & 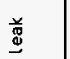 & ㅇ & 웅 & $\stackrel{\text { 帝 }}{-}$ \\
\hline 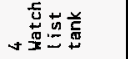 & x & & & & & , & . & . & . & & & . & \\
\hline 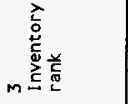 & $\sim$ & 0 & v & 0 & 0 & $\sim$ & 0 & $\sim$ & 0 & 0 & $\checkmark$ & $\checkmark$ & 0 \\
\hline N & $\checkmark$ & . & . & & . & , & . & . & . & . & 0 & ' & . \\
\hline$-\frac{.}{5}$ & $\dot{x}$ & 离을 & $\dot{x} \equiv$ & $\dot{x}$ & $\dot{x}^{\prime}$ & 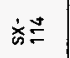 & $\dot{x}=$ & $\therefore \overline{\underline{\underline{O}}}$ & $\therefore \approx$ & $\therefore \stackrel{2}{20}$ & $\therefore$ t & $\therefore$ 농 & $\therefore \stackrel{\circ}{10}$ \\
\hline
\end{tabular}


HNF-SD-WM-CN-105, Rev. 0

\begin{tabular}{|c|c|c|c|c|c|c|c|c|c|c|c|c|}
\hline 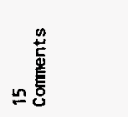 & 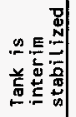 & 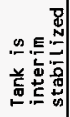 & 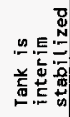 & 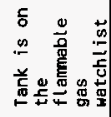 & 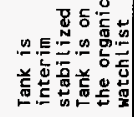 & 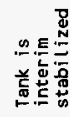 & 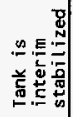 & 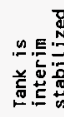 & 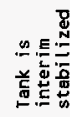 & 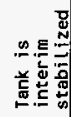 & 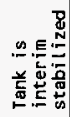 & 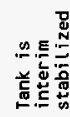 \\
\hline 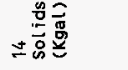 & $\stackrel{R}{=}$ & ఫ & ஸ̊ & 旁 & 号 & 8 & $\stackrel{\infty}{\sim}$ & $\bar{\sim}$ & 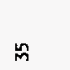 & 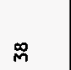 & $\Phi$ & $\hat{N}$ \\
\hline 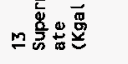 & 0 & 0 & 0 & $m$ & 0 & r & - & 0 & 0 & 0 & $m$ & 0 \\
\hline 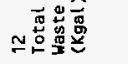 & $\underline{\underline{E}}$ & s & ๓ & 离 & 马̊ & 5 & నे & $\bar{N}$ & m & $\stackrel{\infty}{m}$ & - & $\grave{\sim}$ \\
\hline$=$ & 品 & 品 & n్ & 品 & ถึ & 品 & 品 & 兄 & 出 & 出 & 象 & $\mathfrak{k}$ \\
\hline 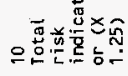 & นุก & 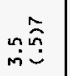 & 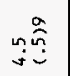 & $\stackrel{n}{\text { ñ. }}$ & $\stackrel{n}{n} \underline{x}$ & น & m & 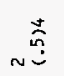 & 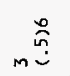 & $\stackrel{\stackrel{0}{n}}{\mathrm{n}}$ & $m \stackrel{0}{n}$ & N \\
\hline 暗昜 & 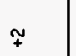 & $\mp$ & $\mp$ & $\mp$ & $\mp$ & 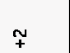 & $\mp$ & $\mp$ & $\mp$ & $\uparrow$ & $\mp$ & 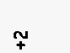 \\
\hline م & $\cong$ & $\cong$ & $\underline{\simeq}$ & 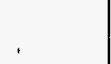 & $\cong$ & $\cong$ & $\cong$ & $\cong$ & $\Omega$ & $\approx$ & $\cong$ & $\cong$ \\
\hline 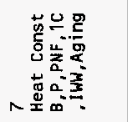 & $\because$ & $\underline{\underline{\prime}}$ & $\cong$ & & & , & . & . & . & , & $\stackrel{0}{\infty}$ & . \\
\hline 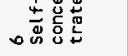 & . & . & & & . & . & , & , & . & & & \\
\hline 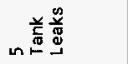 & 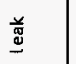 & 总 & 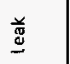 & 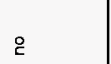 & 菢 & 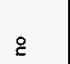 & \& & உ & 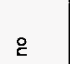 & $\stackrel{2}{q}$ & 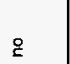 & $\stackrel{8}{\complement}$ \\
\hline 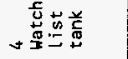 & , & . & . & $x$ & $x$ & . & . & . & . & . & & \\
\hline$M$ & $\sim$ & 0 & $\sim$ & $\checkmark$ & $\checkmark$ & N & $\checkmark$ & $N$ & $\checkmark$ & $\checkmark$ & $N$ & N \\
\hline 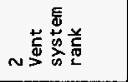 & & . & . & + & 0 & . & & . & . & . & . & \\
\hline - & $\therefore \hat{}$ & 둉 & 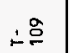 & 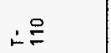 & $\therefore E$ & 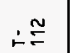 & I- & - ָี & i筩 & - 总 & $\dot{x} \bar{x} \bar{g}$ & $\dot{x}$ \\
\hline
\end{tabular}


HNF-SD-WM-CN-105, Rev. 0

\begin{tabular}{|c|c|c|c|c|c|c|c|c|c|c|c|c|c|}
\hline n & 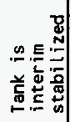 & 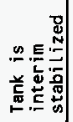 & 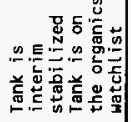 & 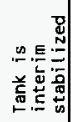 & 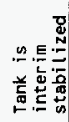 & 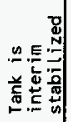 & 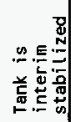 & 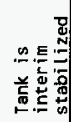 & 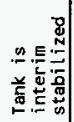 & 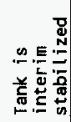 & 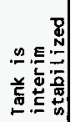 & 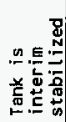 & 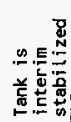 \\
\hline 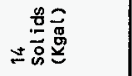 & $\underline{n}$ & t & \%े & 学 & 出 & m & 总 & శ్ & R & 话 & 鸽 & 岗 & 옴 \\
\hline m总密离 & 0 & - & 0 & 0 & - & 0 & 0 & 0 & 0 & 0 & 0 & 0 & 0 \\
\hline 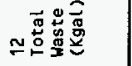 & in & ถి & 今े & 急 & m̊ & m & 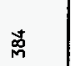 & $\widetilde{\widetilde{q}}$ & $\stackrel{p}{m}$ & वे & $\widehat{8}$ & ผ̂̉ & 옹 \\
\hline 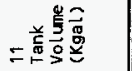 & 㳭 & $\stackrel{\infty}{\kappa}$ & 吕 & $\stackrel{\infty}{\kappa}$ & 垖 & 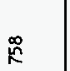 & 吕 & 品 & 员 & $\stackrel{\infty}{\kappa}$ & 员 & 点 & $\stackrel{\infty}{R}$ \\
\hline 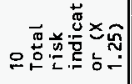 & N & $-\tilde{n}$ & Nָ정 & m气 & $m \stackrel{0}{n}$ & N & ñ & nn & นุำ & $m \stackrel{0}{n}$ & nกำ & min & n气 \\
\hline a气 & ฯ & $\mp$ & $\stackrel{\sim}{+}$ & $\stackrel{\sim}{+}$ & $\dddot{+}$ & 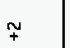 & $\dddot{\mp}$ & $\underset{+}{\Psi}$ & $\underset{+}{\sim}$ & $\dddot{+}$ & 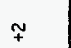 & 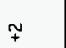 & 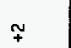 \\
\hline 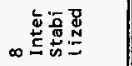 & $\cong$ & $\simeq$ & $\cong$ & $\stackrel{\infty}{=}$ & 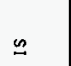 & $\cong$ & $\cong$ & 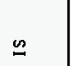 & $\stackrel{\omega}{\omega}$ & $\cong$ & $\cong$ & 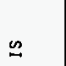 & $\cong$ \\
\hline 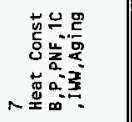 & & . & ' & ' & . & ' & $\underline{\underline{ }}$ & $\underline{.}$ & $\underline{\underline{ }}$ & ' & $\cong$ & 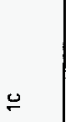 & . \\
\hline 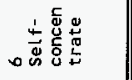 & & ' & , & I & ' & ' & , & , & &. & . & ' & ' \\
\hline 詑美兽 & $\stackrel{8}{\complement}$ & ? & $\stackrel{\text { \% }}{9}$ & ๕ீ & 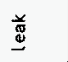 & \& & ๕ & 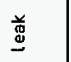 & 울 & 9 & 㡙 & 嵒 & 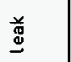 \\
\hline 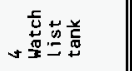 & ' & . & $x$ & ' & , & . & . & I & . & ' & ' & . & ' \\
\hline 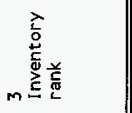 & $\sim$ & 0 & ง & $\checkmark$ & 0 & N & $\sim$ & $\checkmark$ & $\sim$ & $\checkmark$ & $\checkmark$ & $\checkmark$ & $\checkmark$ \\
\hline 〜 & ' & & 0 & . & ' & . & ' & ' & ' & . & ' & ' & , \\
\hline - & $x^{\mathfrak{m}}$ & $\dot{x}$ & $\underset{\searrow}{\searrow}$ & 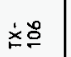 & $\dot{x}$ & $\dot{x}_{x} \stackrel{\infty}{\circ}$ & 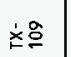 & $\stackrel{x}{x} \stackrel{0}{=}$ & $\dot{x}=$ & $\stackrel{x}{x}$ & $\dot{x} \stackrel{m}{=}$ & $\dot{x}^{ \pm}$ & $\stackrel{\grave{x}}{\unrhd}=$ \\
\hline
\end{tabular}


HNF-SD-WM-CN-105, Rev. 0

\begin{tabular}{|c|c|c|c|c|c|c|c|c|c|c|c|c|}
\hline 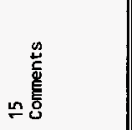 & 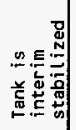 & 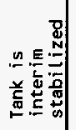 & 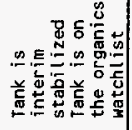 & 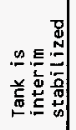 & 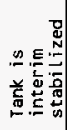 & 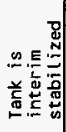 & 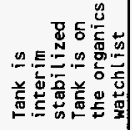 & 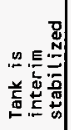 & 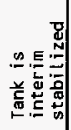 & 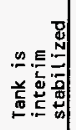 & & 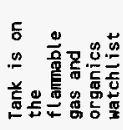 \\
\hline 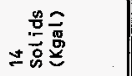 & స్ু & జิ & जे & $\stackrel{\infty}{=}$ & it & 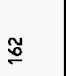 & M & $\bar{\aleph}$ & $\approx$ & $\mathbb{N}$ & 怘 & 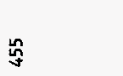 \\
\hline 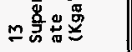 & 0 & 0 & 0 & 0 & o & 0 & $\mathrm{~m}$ & 0 & 0 & m & $\stackrel{\infty}{\simeq}$ & $\stackrel{m}{2}$ \\
\hline 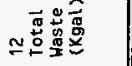 & $\bar{్}$ & ะู้ & जे & $\stackrel{\infty}{=}$ & st & $\cong$ & $\underset{\sim}{0}$ & $\bar{N}$ & $\simeq$ & $\stackrel{\sim}{\sim}$ & 雍 & 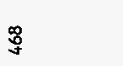 \\
\hline 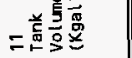 & $\stackrel{\infty}{\kappa}$ & א๊ & 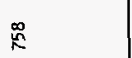 & 点 & 吕 & $\stackrel{\infty}{R}$ & 唯 & $\stackrel{\infty}{R}$ & 品 & ถึ่ & 总 & हి \\
\hline 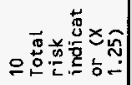 & nก & $\min _{n}^{\bar{n}}$ & $\frac{m}{m}$ & นุ้ & $\stackrel{m}{n}$ & 该 & 哭态 & $\stackrel{\infty}{\stackrel{0}{n}}$ & m气̊ & m气̆ & 0 & $\stackrel{\infty}{\underline{m}} \stackrel{x}{=}$ \\
\hline 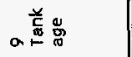 & $\underset{+}{\tau}$ & $\stackrel{\sim}{+}$ & $\dddot{\tau}$ & $\underset{+}{\sim}$ & $\stackrel{\sim}{+}$ & $\mp$ & $\underset{+}{\sim}$ & $\underset{+}{\sim}$ & $\dddot{+}$ & $\mp$ & $\uparrow$ & $\simeq$ \\
\hline 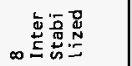 & 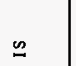 & $\approx$ & $\approx$ & $\stackrel{\sim}{\sim}$ & $\approx$ & 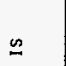 & $\cong$ & $\stackrel{\infty}{\sim}$ & $\cong$ & $\stackrel{\infty}{\sim}$ & . & , \\
\hline 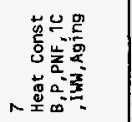 & , & $\underline{-}$ & $\underline{ }$ & $\underline{\underline{ }}$ & $\underline{\underline{ }}$ & $\underline{\square}$ & $\underline{U}$ & ' & . & . & ' & $\frac{u}{\frac{L}{a}}$ \\
\hline 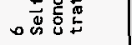 & , & . & ' & ' & . & , & . & ' & ' & ' & ' & ' \\
\hline 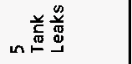 & 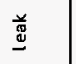 & 嵒 & 울 & 总 & 욷 & 若 & 单 & 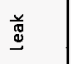 & 莺 & 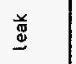 & $\mathrm{g}$ & \& \\
\hline 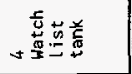 & . & . & $\star$ & . & . & & $\times$ & & . & . & & $\times$ \\
\hline 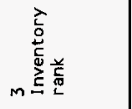 & $\checkmark$ & + & $\sim$ & $N$ & 0 & $\sim$ & 0 & N & 0 & 0 & $\checkmark$ & $\checkmark$ \\
\hline 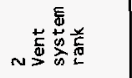 & . & , & 0 & ' & ' & & 0 & . & ' & . & . & $\checkmark$ \\
\hline 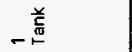 & $\dot{x} \underset{x}{ }$ & 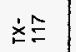 & 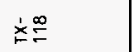 & خ் & $亡 \cong$ & خั? & $\frac{\square}{\geqslant}$ & 눈음 & 之气 & $j \Phi$ & ذ) & j) \\
\hline
\end{tabular}




\begin{tabular}{|c|c|c|c|c|c|c|c|c|c|c|c|c|c|c|}
\hline 1 Tank & $\begin{array}{l}2 \\
\text { Vent } \\
\text { system } \\
\text { rank }\end{array}$ & $\begin{array}{l}3 \\
\text { Inventory } \\
\text { rank }\end{array}$ & $\begin{array}{l}4 \\
\text { Watch } \\
\text { list } \\
\text { tank }\end{array}$ & $\begin{array}{l}5 \\
\text { Jank } \\
\text { Leaks }\end{array}$ & $\begin{array}{l}6 \\
\text { Self- } \\
\text { concen } \\
\text { trate }\end{array}$ & $\begin{array}{l}7 \\
\text { Heat Const } \\
\text { B,P,PNF, IC } \\
\text {, Iw, Aging }\end{array}$ & $\begin{array}{l}8 \\
\text { Inter } \\
\text { stabi } \\
\text { lized }\end{array}$ & $\begin{array}{l}9 \\
\text { Tank } \\
\text { age }\end{array}$ & $\begin{array}{l}10 \\
\text { Total } \\
\text { risk } \\
\text { indicat } \\
\text { or }(X X \\
1.25)\end{array}$ & $\begin{array}{l}11 \\
\text { Tank } \\
\text { Volume } \\
\text { (Kgal) }\end{array}$ & $\begin{array}{l}12 \\
\text { Total } \\
\text { Waste } \\
\text { (Kgal) }\end{array}$ & $\begin{array}{l}13 \\
\text { Supern } \\
\text { ate } \\
\text { (Kgal) }\end{array}$ & $\begin{array}{l}14 \\
\text { Sol ids } \\
\text { (Kgal) }\end{array}$ & $\begin{array}{l}15 \\
\text { Comments }\end{array}$ \\
\hline $\begin{array}{l}u- \\
104\end{array}$ & - & 2 & - & leak & - & - & Is & +2 & $4^{4}(.5) 8$ & 530 & 122 & 0 & 122 & $\begin{array}{l}\text { Tank is } \\
\text { interim } \\
\text { stabilized }\end{array}$ \\
\hline $\begin{array}{l}U- \\
105\end{array}$ & 4 & 4 & $x$ & no & $\cdot$ & - & - & +2 & $\begin{array}{l}12.5 \\
10 x\end{array}$ & 530 & 418 & 37 & 381 & $\begin{array}{l}\text { Tank is on } \\
\text { the } \\
\text { flatrmable } \\
\text { gas and } \\
\text { organics } \\
\text { watchlist }\end{array}$ \\
\hline $\begin{array}{l}\text { U- } \\
106\end{array}$ & 0 & 2 & $x$ & no & - & - & . & +2 & $\begin{array}{l}5 \\
4 x\end{array}$ & 530 & 226 & 15 & 211 & $\begin{array}{l}\text { Tank is on } \\
\text { the organics } \\
\text { watchl ist }\end{array}$ \\
\hline $\begin{array}{l}\mathrm{U-} \\
107\end{array}$ & 4 & 4 & $x$ & no & - & - & - & +2 & $\begin{array}{l}12.5 \\
10 x\end{array}$ & 530 & 406 & 31 & 375 & $\begin{array}{l}\text { Tank is on } \\
\text { the } \\
\text { flammable } \\
\text { gas and } \\
\text { organics } \\
\text { watchlist }\end{array}$ \\
\hline $\begin{array}{l}\text { U- } \\
108\end{array}$ & 4 & 4 & $x$ & no & $\cdot$ & - & $\cdot$ & +2 & 12.5 & 530 & 468 & 24 & 444 & $\begin{array}{l}\text { Tank is on } \\
\text { the } \\
\text { flammable } \\
\text { gas } \\
\text { watchlist }\end{array}$ \\
\hline $\begin{array}{l}\text { U- } \\
\text { t09 }\end{array}$ & 4 & 4 & $x$ & no & - & PNF & - & +2 & $\begin{array}{l}13.8 \\
11 x\end{array}$ & 530 & 463 & 19 & 444 & $\begin{array}{l}\text { Tank is on } \\
\text { the } \\
\text { flammable } \\
\text { gas } \\
\text { watch } 1 \text { ist }\end{array}$ \\
\hline $\begin{array}{l}\mathbf{U}- \\
110\end{array}$ & - & 2 & - & leak & boil & $1 \mathrm{C}$ & is & +2 & $\frac{6}{(.5) 12}$ & 530 & 186 & 0 & 186 & $\begin{array}{l}\text { Tank is } \\
\text { interim } \\
\text { stabilized }\end{array}$ \\
\hline $\begin{array}{l}U- \\
111\end{array}$ & 0 & 4 & $x$ & no & - & PNF, 1C & - & +2 & $\begin{array}{l}10 \\
8 x\end{array}$ & 530 & 329 & 0 & 329 & $\begin{array}{l}\text { Tank is on } \\
\text { the organics } \\
\text { watchl ist }\end{array}$ \\
\hline $\begin{array}{l}\mathrm{U}- \\
112\end{array}$ & $\cdot$ & 0 & - & leak & $\cdot$ & 10 & is & +2 & $\begin{array}{l}3.5 \\
(.5) 7\end{array}$ & 530 & 49 & 4 & 45 & $\begin{array}{l}\text { Tank is } \\
\text { interim } \\
\text { stabilized }\end{array}$ \\
\hline $\begin{array}{l}U- \\
201\end{array}$ & - & 2 & - & no & - & - & IS & +2 & $\begin{array}{l}2 \\
6.5) 4\end{array}$ & 55 & 5 & 1 & 4 & $\begin{array}{l}\text { Tank is } \\
\text { interim } \\
\text { stabilized }\end{array}$ \\
\hline $\begin{array}{l}\text { U- } \\
202\end{array}$ & - & 2 & - & no & - & - & Is & +2 & $2.5) 4$ & 55 & 5 & 1 & 4 & $\begin{array}{l}\text { Tank is } \\
\text { interim } \\
\text { stabilized }\end{array}$ \\
\hline
\end{tabular}


HNF-SD-WM-CN-105, Rev. 0

\begin{tabular}{|c|c|c|c|c|c|c|c|c|c|c|c|c|}
\hline 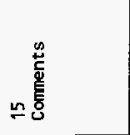 & 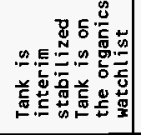 & 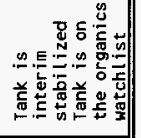 & & & & 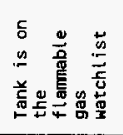 & 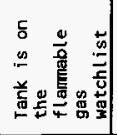 & 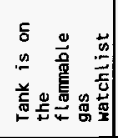 & & & & \\
\hline 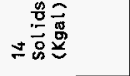 & $\sim$ & N & & m & ळ. & 人̂ & 呄 & 0 & $=$ & $\hat{\mathbb{N}}$ & 0 & 0 \\
\hline m & - & - & & ๓ & \& & $\stackrel{\alpha}{q}$ & $\bar{\alpha}$ & $\stackrel{\text { ̊̊ }}{=}$ & 今 & 產 & $\stackrel{9}{0}$ & ò \\
\hline 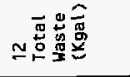 & $m$ & m & & $\cong$ & 号 & ณू & 㟧 & $\stackrel{\infty}{\stackrel{\Im}{\leftrightarrows}}$ & $\stackrel{\circ}{\circ}$ & 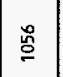 & 管 & $\underline{\underline{s}}$ \\
\hline 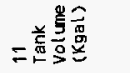 & in & in & & $\stackrel{\circ}{\stackrel{\circ}{\circ}}$ & $\stackrel{\circ}{=}$ & $\stackrel{\mathscr{8}}{\stackrel{8}{\circ}}$ & $\stackrel{8}{!}$ & $\stackrel{8}{\circ}$ & $\stackrel{\circ}{\circ}$ & $\stackrel{8}{=}$ & $\stackrel{8}{\circ}$ & $\stackrel{8}{\circ}$ \\
\hline 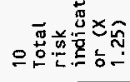 & 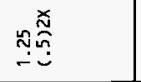 & 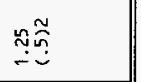 & & n & $a$ & $\stackrel{m}{=} \times$ & $\stackrel{m}{=} \times$ & $\stackrel{m}{=} \underset{x}{\sigma}$ & r & $a$ & $a$ & a \\
\hline م. & $\approx$ & $\cong$ & & 0 & 0 & 0 & 0 & 0 & 。 & 。 & 0 & 0 \\
\hline 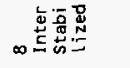 & $\cong$ & $\Leftrightarrow$ & & & . & & . & . & . & . & . & . \\
\hline 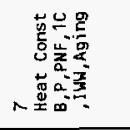 & & ' & & , & ' & , & ' & . & ' & , & , & ' \\
\hline 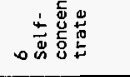 & & ' & & ' & . & . & ' & . & , & . & . & ' \\
\hline 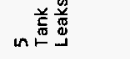 & உ & ๕ & & ㅇ & ¿ & $\stackrel{8}{\varepsilon}$ & $\stackrel{\circ}{ }$ & உ & \& & ๕̊ & $\stackrel{8}{\varepsilon}$ & 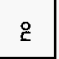 \\
\hline 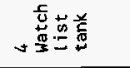 & $x$ & $\times$ & & . & , & $x$ & $\times$ & $\times$ & . & , & & \\
\hline 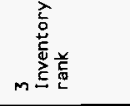 & 0 & 0 & 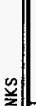 & $N$ & $\checkmark$ & ง & $\checkmark$ & \pm & $N$ & 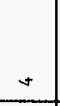 & + & + \\
\hline 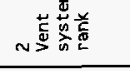 & 0 & 0 & 言 & in & in & n & in & in & in & n & n & in \\
\hline 恙 & 塔 & 㳳 & 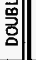 & 交司 & $\sum \frac{1}{x}=$ & $\dot{z} \frac{\dot{m}}{2}$ & 安吉 & 主奚 & 웡 & 妾包 & 워 & 定疍 \\
\hline
\end{tabular}




\begin{tabular}{|c|c|c|c|c|c|c|c|c|c|c|c|c|c|c|}
\hline $\begin{array}{l}1 \\
\text { Tank }\end{array}$ & $\begin{array}{l}2 \\
\text { Vent } \\
\text { system } \\
\text { rank }\end{array}$ & $\begin{array}{l}3 \\
\text { Inventory } \\
\text { rank }\end{array}$ & $\begin{array}{l}4 \\
\text { Watch } \\
\text { list } \\
\text { tank }\end{array}$ & $\begin{array}{l}5 \\
\text { Tank } \\
\text { Leaks }\end{array}$ & $\begin{array}{l}6 \\
\text { Self- } \\
\text { concen } \\
\text { trate }\end{array}$ & $\begin{array}{l}7 \\
\text { Heat Const } \\
\text { B,P,PNF, IC } \\
\text {, iw , Aging }\end{array}$ & $\begin{array}{l}8 \\
\text { Inter } \\
\text { stabi } \\
\text { Lized }\end{array}$ & $\begin{array}{l}\stackrel{9}{\text { Tank }} \\
\text { age }\end{array}$ & $\begin{array}{l}10 \\
\text { Ootal } \\
\text { risk } \\
\text { indicat } \\
\text { or }(x \\
1.25) \\
\end{array}$ & $\begin{array}{l}11 \\
\text { Tank } \\
\text { Volume } \\
\text { (Kgal) }\end{array}$ & $\begin{array}{l}12 \\
\text { Total } \\
\text { Waste } \\
\text { (Kgal) }\end{array}$ & $\begin{array}{l}13 \\
\text { Supern } \\
\text { ate } \\
\text { (Kgal) }\end{array}$ & $\begin{array}{l}14 \\
\text { Sol ids } \\
\text { (Kgal) }\end{array}$ & $\begin{array}{l}15 \\
\text { Comments }\end{array}$ \\
\hline $\begin{array}{l}\text { AP- } \\
103 \\
\end{array}$ & 5 & 0 & - & no & - & - & - & 0 & 5 & 1160 & 23 & 22 & 1 & \\
\hline $\begin{array}{l}\text { AP- } \\
104\end{array}$ & 5 & 0 & - & no & $\cdot$ & - & - & 0 & 5 & 1160 & 26 & 26 & 0 & \\
\hline $\begin{array}{l}\text { AP- } \\
105 \\
\end{array}$ & 5 & 4 & - & no & - & - & - & 0 & 9 & 1160 & 1123 & 969 & 154 & \\
\hline $\begin{array}{l}\text { AP- } \\
106 \\
\end{array}$ & 5 & 2 & - & no & - & - & - & 0 & 7 & 1160 & 262 & 262 & 0 & \\
\hline $\begin{array}{l}\text { AP - } \\
10 ?\end{array}$ & 5 & 0 & - & no & $\cdot$ & - & - & 0 & 5 & 1160 & 21 & 21 & 0 & \\
\hline $\begin{array}{l}\text { AP- } \\
108 \\
\end{array}$ & 5 & 0 & - & no & - & - & - & 0 & 5 & 1160 & 46 & 46 & 0 & \\
\hline $\begin{array}{l}\text { AW- } \\
101\end{array}$ & 5 & 4 & $x$ & no & $\cdot$ & . & - & +1 & $\begin{array}{l}12.5 \\
10 x\end{array}$ & 1160 & 1128 & 1044 & 84 & $\begin{array}{l}\text { Iank is on } \\
\text { the } \\
\text { flammable } \\
\text { gas } \\
\text { watchlist }\end{array}$ \\
\hline $\begin{array}{l}\text { AN- } \\
102 \\
\end{array}$ & 5 & 0 & - & no & - & - & - & +1 & 6 & 1160 & 99 & 63 & 36 & \\
\hline $\begin{array}{l}\text { AH- } \\
103 \\
\end{array}$ & 5 & 2 & $\cdot$ & no & - & - & - & +1 & 8 & 1160 & 514 & 151 & 363 & . \\
\hline $\begin{array}{l}\text { AH- } \\
104\end{array}$ & 5 & 4 & - & no & - & . & - & +1 & 10 & 1160 & 1121 & 831 & 290 & \\
\hline $\begin{array}{l}\text { AH- } \\
105 \\
\end{array}$ & 5 & 2 & - & no & - & - & - & +1 & 8 & 1160 & 440 & 160 & 280 & \\
\hline $\begin{array}{l}\text { AW- } \\
106\end{array}$ & 5 & 2 & - & no & $\cdot$ & - & - & +1 & 8 & 1160 & 321 & 96 & 225 & \\
\hline $\begin{array}{l}\text { AY- } \\
101 \\
\end{array}$ & 5 & 4 & - & no & - & $\begin{array}{l}\text { B,P2, } \\
\text { Aging }\end{array}$ & - & +2 & 14 & 1000 & 918 & 824 & 94 & \\
\hline $\begin{array}{l}\text { AY- } \\
102\end{array}$ & 5 & 4 & - & no & $\cdot$ & Aging & - & +2 & 12 & 1000 & 841 & 811 & 30 & \\
\hline $\begin{array}{l}\text { AZ- } \\
101\end{array}$ & 5 & 4 & - & no & - & $\begin{array}{l}\text { Aging, } \\
\text { P3 }\end{array}$ & - & +1 & 12 & 1000 & 870 & 835 & 35 & \\
\hline $\begin{array}{l}\text { AZ- } \\
102\end{array}$ & 5 & 4 & - & no & - & $\begin{array}{l}\text { Aging, } \\
\text { P3 }\end{array}$ & - & +1 & 12 & 1000 & 922 & 827 & 95 & \\
\hline
\end{tabular}




\begin{tabular}{|c|c|c|c|c|c|c|c|c|c|c|c|c|c|c|}
\hline $\begin{array}{l}1 \\
\text { Tank }\end{array}$ & $\begin{array}{l}2 \\
\text { vent } \\
\text { system } \\
\text { rank }\end{array}$ & $\begin{array}{l}3 \\
\text { Inventory } \\
\text { rank }\end{array}$ & $\begin{array}{l}4 \\
\text { Watch } \\
\text { list } \\
\text { tank }\end{array}$ & $\begin{array}{l}5 \\
\text { Tank } \\
\text { Leaks }\end{array}$ & $\begin{array}{l}6 \\
\text { Self- } \\
\text { concen } \\
\text { trate }\end{array}$ & $\begin{array}{l}7 \\
\text { Heat Const } \\
\text { B,P,PNF, 1C } \\
\text {, IWW, Aging }\end{array}$ & $\begin{array}{l}8 \\
\text { Inter } \\
\text { Stabi } \\
\text { lized }\end{array}$ & $\begin{array}{l}9 \\
\text { Tank } \\
\text { age }\end{array}$ & $\begin{array}{l}10 \\
\text { Total } \\
\text { risk } \\
\text { indicat } \\
\text { or }(x \\
1.25) \\
\end{array}$ & $\begin{array}{l}11 \\
\text { Tank } \\
\text { Volume } \\
\text { (Kgal) }\end{array}$ & $\begin{array}{l}12 \\
\text { Total } \\
\text { Waste } \\
\text { (Kgal) }\end{array}$ & $\begin{array}{l}13 \\
\text { Supern } \\
\text { ate } \\
\text { (Kgal) }\end{array}$ & $\begin{array}{l}14 \\
\text { Sol ids } \\
\text { (Kgal) }\end{array}$ & $\begin{array}{l}15 \\
\text { Comments }\end{array}$ \\
\hline $\begin{array}{l}\text { SY- } \\
101\end{array}$ & 5 & 4 & $x$ & no & - & PNF & - & +2 & $\begin{array}{l}15 \\
12 x\end{array}$ & 1160 & 1114 & 1073 & 41 & $\begin{array}{l}\text { Tank is on } \\
\text { the } \\
\text { flammable } \\
\text { gas } \\
\text { watchl ist }\end{array}$ \\
\hline $\begin{array}{l}\text { sy- } \\
102\end{array}$ & 5 & 4 & - & no & - & PNF & & +2 & 12 & 1160 & 610 & 539 & 71 & \\
\hline $\begin{array}{l}\text { SY- } \\
103\end{array}$ & 5 & 4 & $x$ & no & - & - & - & +2 & $\begin{array}{l}13.8 \\
11 x\end{array}$ & 1160 & 748 & 382 & 366 & $\begin{array}{l}\text { Tank is on } \\
\text { the } \\
\text { flarmable } \\
\text { gas } \\
\text { watch! ist }\end{array}$ \\
\hline
\end{tabular}


Key for Table A-1

(2) a. A dash (-) in the vent system rank column indicates that the tank is 'interim stabilized' and not a watch-list tank, so the vent system status is not a great concern.

b. A zero (0) in the vent system rank column indicates that the tank is a watch-list tank but it is not on the flammable gas watchlist, so the vent system status is not a great concern.

c. A four (4) in the vent system rank column indicates that the tank has a passive breathing system and it is on the watch list for flammable gas buildup.

d. A five (5) in the vent system rank column indicates that the tank normally has an operating, active ventilation system, and that the system should be returned to service as soon as possible, if shutdown due to a natural phenomena event, to maintain cooling and confinement.

(3) The inventory ranking is based on the following waste volumes:

Rank $4=\geq 50 \%$ tank full

Rank $2=10-49.9 \%$ tank ful1

Rank $0=<10 \%$ tank full

(4) An ' $X$ ' in this column indicates the tank is on a watch 1 ist.

(5) Tank leaks: Tank is a known leaker.

(6) Self-concentrator: the tanks indicating 'boil' were allowed to boil as a means of cooling, this only includes SSTs.

(7) Heat Const.: this column indicates that waste constituents that were very hot thermally were put into this tank. Symbols include:

Aging: aging waste

$B$ : B-Plant high level waste

P: PUREX high level waste

PNF: Partial neutralized waste

1C: First cycle waste

IWW: PUREX \#1 acid concentrator waste

(8) Interim Stabilization: An 'IS' in this column indicates the tank has been interim stabilized. This process includes pumping most of the liquid out of the tank so that the tank has less than 50,000 gallons of drainable interstitial liquid and less than 5,000 gallons of supernate 1 iquid.

(9) Tank age: a ranking was assigned to each tank according to its age, as follows:

+2 - the tank was built in the 1940 s or 1950 s, it is $40-50$ years old,

+1 - the tank was built in the 1960s or 1970s, it is 20-30 years old,

0 - the tank was built since 1980 . 
(10) The total risk indicator column is a sum of the values in columns 38. The ' $X$ ' in column 5 indicates the tank is on a watchlist. These values include a multiplier of 1.25 to account for the watchlist concerns. The 'IS' in column 9 indicates the tank has been interim stabilized, the risk indicator for these tanks has been multiplied by 0.5 to show the decreased safety risk due to the interim stabilization efforts.

(11) - (14) Inventory of the tank:

(11) Tank total volume, design capacity

(12) Total waste volume in the tank, liquids and solids

(13) Supernate (liquids) volume in the tank

(14) Solids volume in the tank 
HNF-SD-WM-CN-105, Rev. 0

\section{APPENDIX B}

\section{Tank Farm Structural Inspection Priority}


HNF-SD-WM-CN-105, Rev. 0

Table B-1. Tank Farm Structural Inspection Priority

\begin{tabular}{||c|c|c|c|c||}
\hline PRIORITY & TANK FARM & $\begin{array}{c}\text { TANK FARM } \\
\text { TOTAL RISK } \\
\text { INDICATORS }\end{array}$ & $\begin{array}{c}\text { NUMBER OF } \\
\text { TANKS IN } \\
\text { FARM }\end{array}$ & $\begin{array}{c}\text { AVERAGE } \\
\text { TOTAL RISK } \\
\text { INDICATOR }\end{array}$ \\
\hline \hline 1 & SY & 40.8 & 3 & 13.6 \\
\hline 2 & AY & 26 & 2 & 13.0 \\
\hline 3 & AZ & 24 & 2 & 12.0 \\
\hline 4 & SX & 151 & 15 & 10.0 \\
\hline 5 & AN & 65.9 & 7 & 9.41 \\
\hline 6 & AW & 52.5 & 6 & 8.75 \\
\hline 7 & AX & 35 & 4 & 8.75 \\
\hline 8 & A & 46 & 6 & 7.67 \\
\hline 9 & S & 90.1 & 12 & 7.51 \\
\hline 10 & U & 109 & 16 & 6.82 \\
\hline 11 & AP & 54 & 8 & 6.75 \\
\hline 12 & BY & 55 & 12 & 4.58 \\
\hline 13 & T & 73 & 16 & 4.56 \\
\hline 14 & B & 66.4 & 16 & 4.15 \\
\hline 15 & C & 65.1 & 16 & 4.07 \\
\hline 16 & TY & 21.9 & 6 & 3.65 \\
\hline 17 & TX & 65.4 & 18 & 3.63 \\
\hline 18 & BX & 34 & 12 & 2.83 \\
\hline \hline
\end{tabular}




\section{CHECKLIST FOR PEER REVIEW}

Document Reviewed: HNF-SD-WM-CN-105, Rev. 0, Post Natural Phenomena Event Priorities For Structural Investigations In The Tank Farms

Scope of Review: reviwed report but did not verfy references

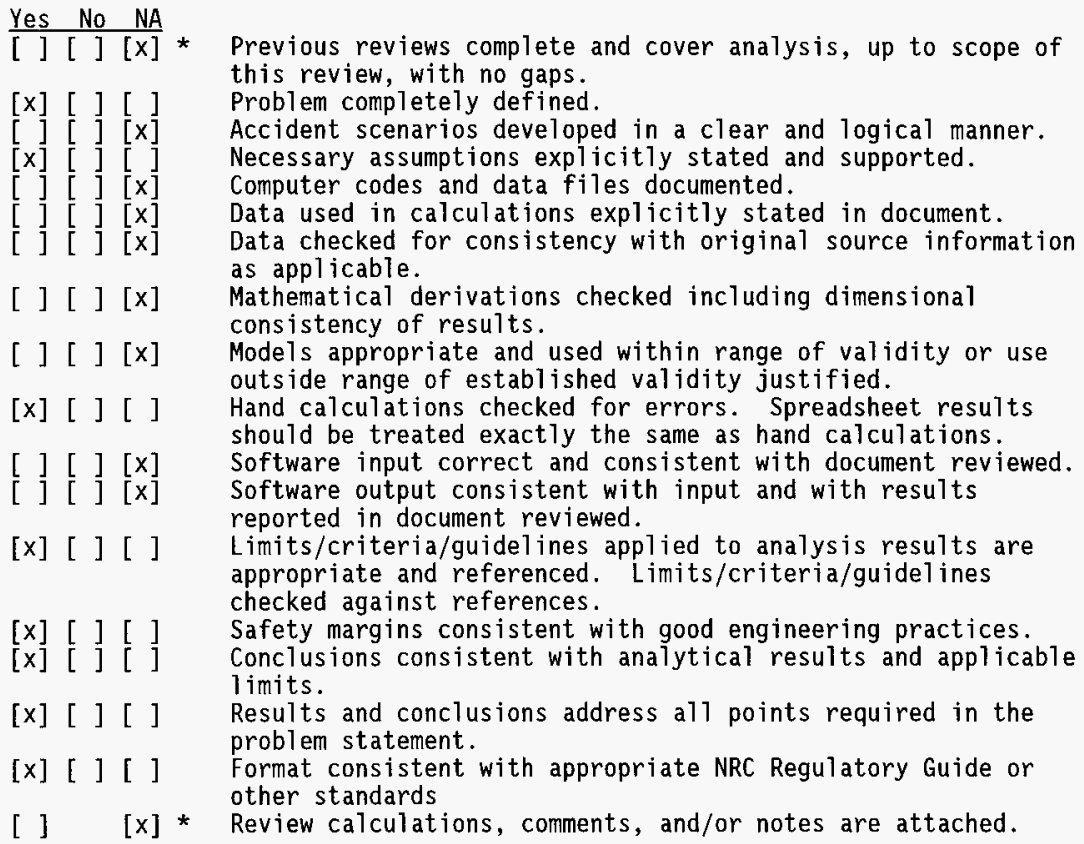

[x] [ ] [ ] Document approved.

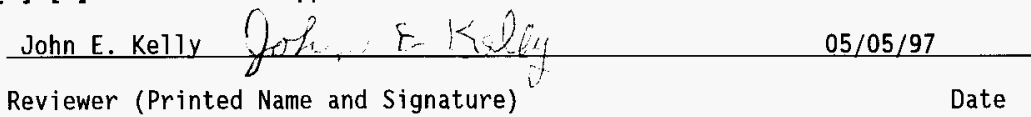

* Any calculations, comments, or notes generated as part of this review should be signed, dated and attached to this checklist. Such material should be labeled and recorded in such a manner as to be intelligible to a technically qualified third party. 